\title{
Development of $\mathrm{Cu}^{2+}$-Based Distance Methods and
}

\author{
Force Field Parameters for the Determination of
}

\section{PNA Conformations and Dynamics by EPR and MD}

\section{Simulations}

Austin Gamble Jarvi ${ }^{1}$, Artur Sargun ${ }^{2}$, Xiaowei Bogetti ${ }^{1}$, Junmei Wang ${ }^{3 *}$, Catalina Achim²*, Sunil Saxena $^{1 *}$

${ }^{1}$ Department of Chemistry, University of Pittsburgh, Pittsburgh, PA 15260, USA

${ }^{2}$ Department of Chemistry, Carnegie Mellon University, Pittsburgh, PA 15213, United States

${ }^{3}$ Department of Pharmaceutical Sciences, University of Pittsburgh, Pittsburgh, PA 15206, United States 


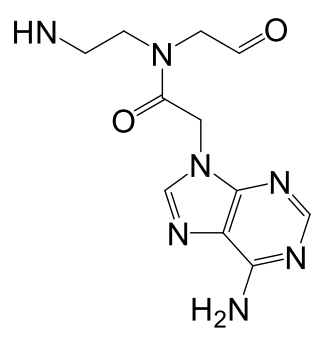

APN

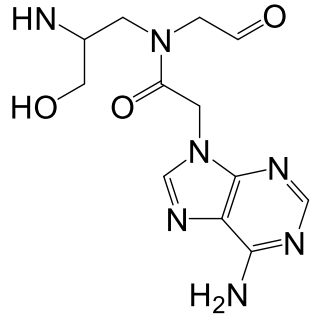

APR

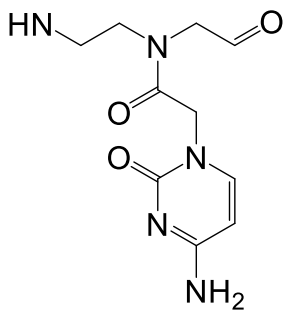

CPN<smiles>Nc1ccn(CC(=O)N(CC=O)CC(N)CO)c(=O)n1</smiles>

CPR
$\mathrm{HN}$<smiles>CCCN(CC=O)C(=O)Cn1cnc2c(=O)[nH]c(N)nc21</smiles>

GPN<smiles>NCCN(CC=O)C(=O)Cc1ccnc2c(O)cccc12</smiles>

QPC/QPN<smiles>NNC(CO)CN(CC=O)C(=O)Cn1cnc2c(=O)[nH]c(N)nc21</smiles>

GPR<smiles>Cc1cn(CC(=O)N(CC=O)CCN)c(=O)[nH]c1=O</smiles>

TPN<smiles>Cc1cn(CC(=O)N(CC=O)CC(N)CO)c(=O)[nH]c1=O</smiles>

TPR

Figure S1. Chemical structures of PNA residues and the model compound of $\mathrm{CuQ}_{2}$. For the $\mathrm{CuQ}_{2}$ model compound (QPC/QPN), the N-termini are capped with acetyl group (red) and the C-termini are capped with N-methyl amide group (blue). It is noted that the QPC and QPN residues have the same chemical structures and we used different atom types for the pyridine nitrogen and phenol oxygen in QPC and QPN so that we can more accurately describe the $\mathrm{CuQ}_{2}$ structure. 

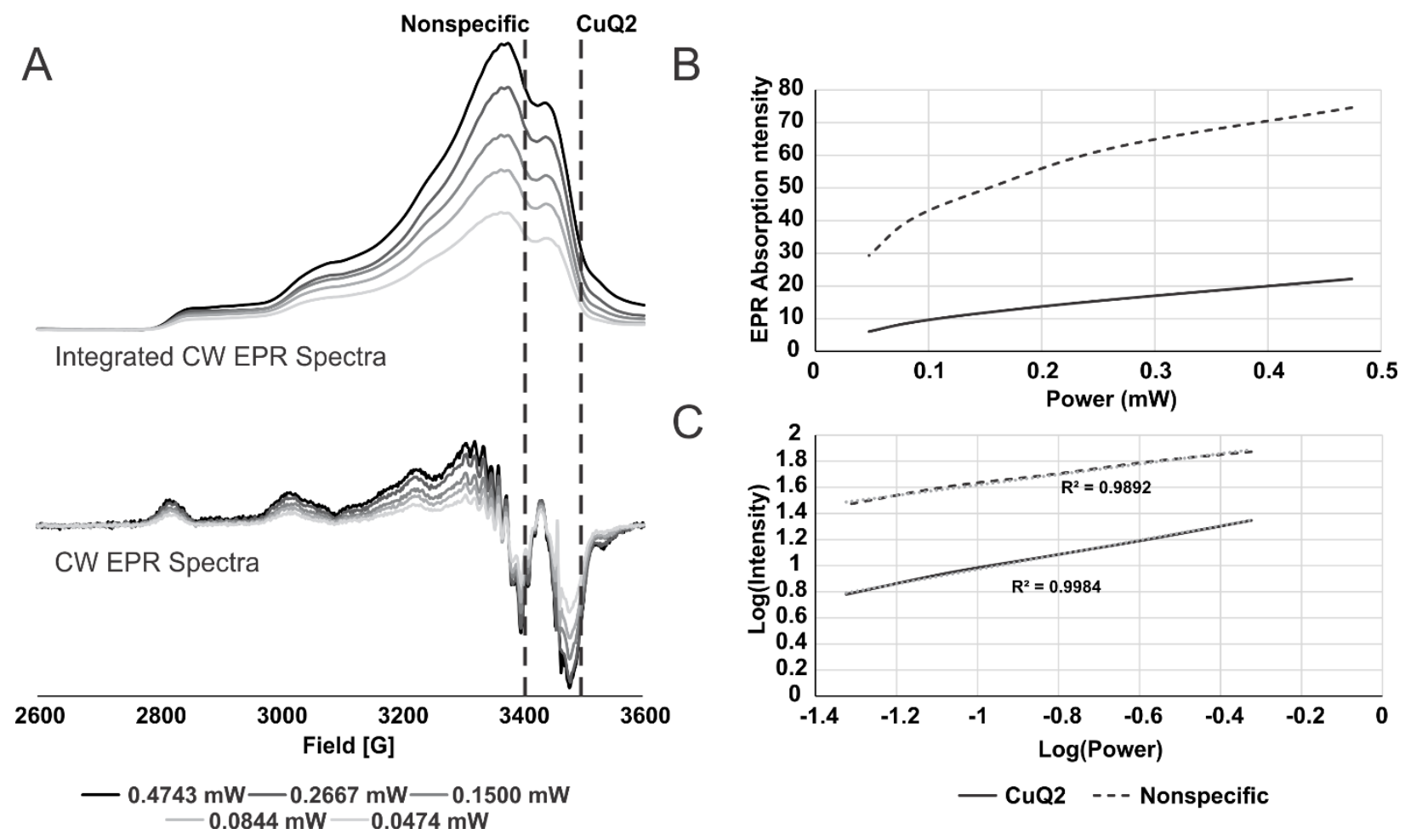

C

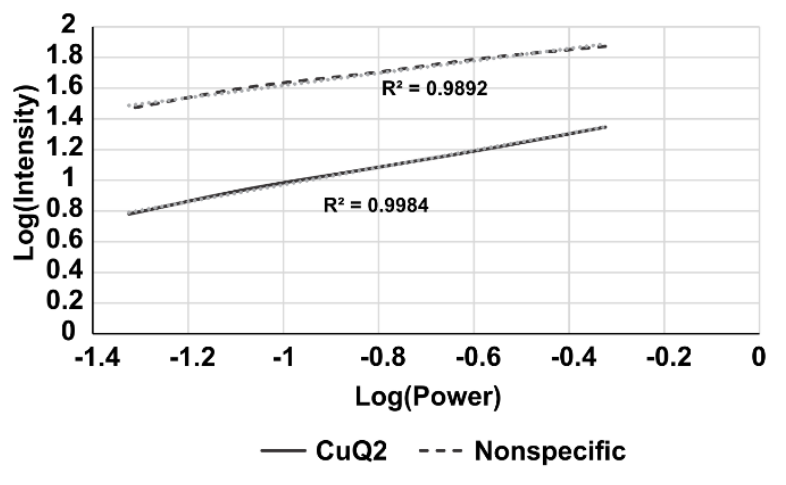

Figure S2. A) CW EPR spectra collected at five microwave power levels to test for sample saturation. The below spectra show the background corrected CW EPR spectra, while the above shows the integration of those spectra in order to extract the absorption intensity. B) Saturation curve examining power against the intensity of absorption. Saturation was monitored at two different magnetic fields shown in (A) that correspond to an area of the spectrum in which each component dominates (cf. Fig. 2). C) $\log _{10}$ plot of the power vs. the EPR intensity. Linear regression on each curve was performed, with $\mathrm{R}^{2}$ values shown for each field monitored. In unsaturated conditions, the intensity of the EPR spectra should increase proportional to the square root of the power. The linear $\log _{10}$ curve indicates that at the power levels used in this work, the $\mathrm{Cu}^{2+}$ species are not being saturated. Experiments were performed on the $\gamma$-PNA with experimental parameters as described in Methods, with the exception of the microwave power applied, which is denoted here. 

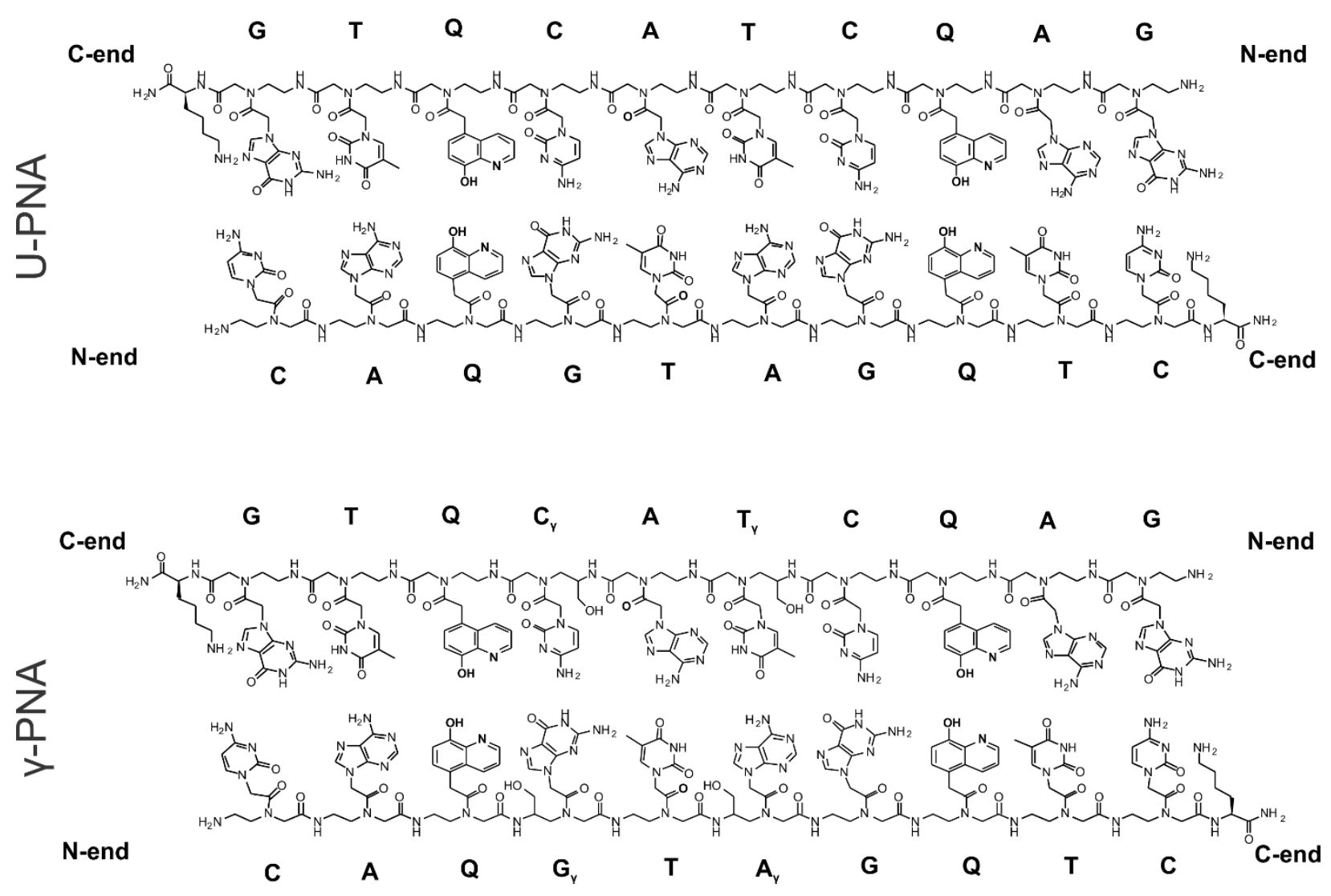

Figure S3. Full chemical structures of the U-PNA (top) and $\gamma$-PNA (bottom).
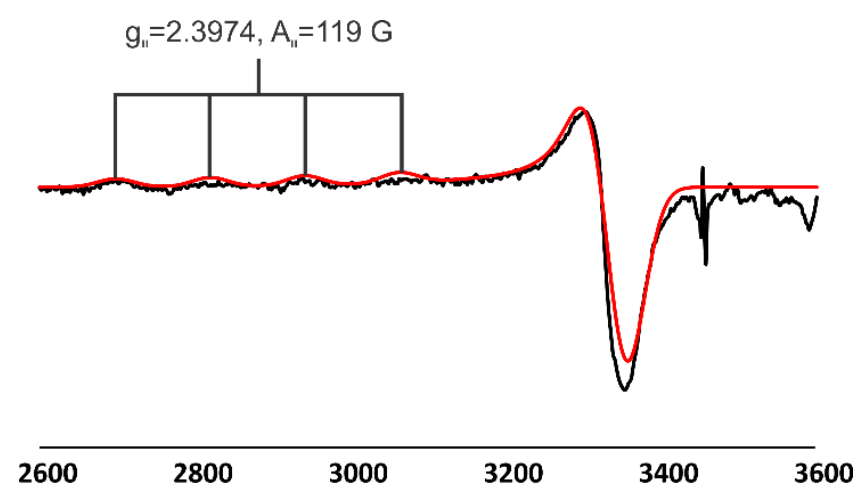

Field [G]

Figure S4. CW EPR spectrum of $150 \mu \mathrm{M} \mathrm{CuCl}_{2}$ in $10 \mathrm{mM}$ sodium phosphate buffer at $\mathrm{pH} 7.0$ (black line), as used in all PNA samples. The simulated spectrum (red line) displayed $\mathrm{g}_{\|}$and $\mathrm{A}_{\|}$ parameters are listed above to emphasize their distinct difference from those of the bound PNA $\mathrm{CuQ}_{2}$ parameters. 

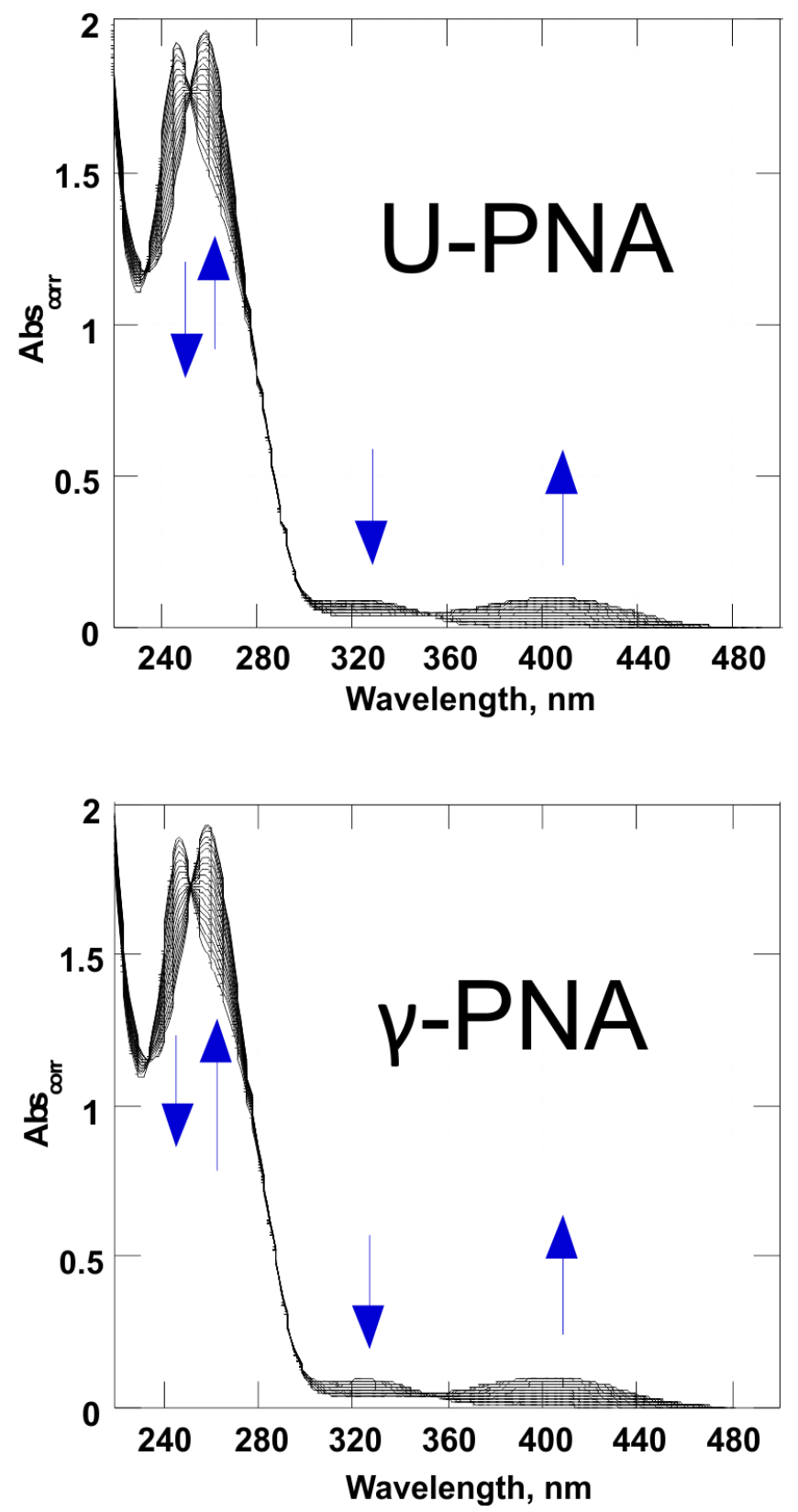

Figure S5. UV/Vis spectra for the titrations of $\mathrm{CuCl}_{2}$ into U-PNA (top) and $\gamma$-PNA (bottom). Arrows indicate the four monitored wavelengths and their trend (increasing or decreasing) with the addition of $\mathrm{CuCl}_{2}$. 


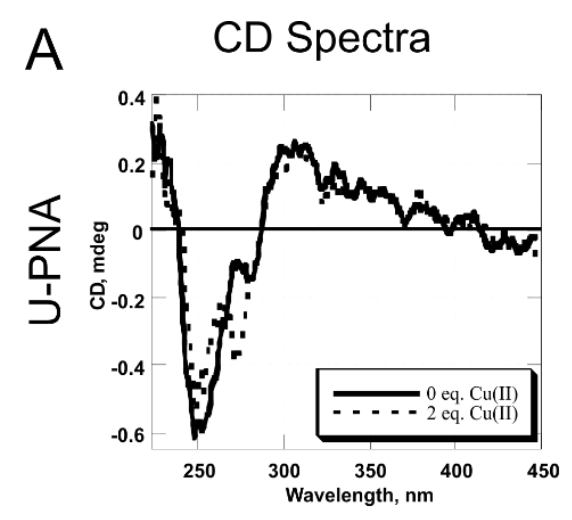

C Melting Curves
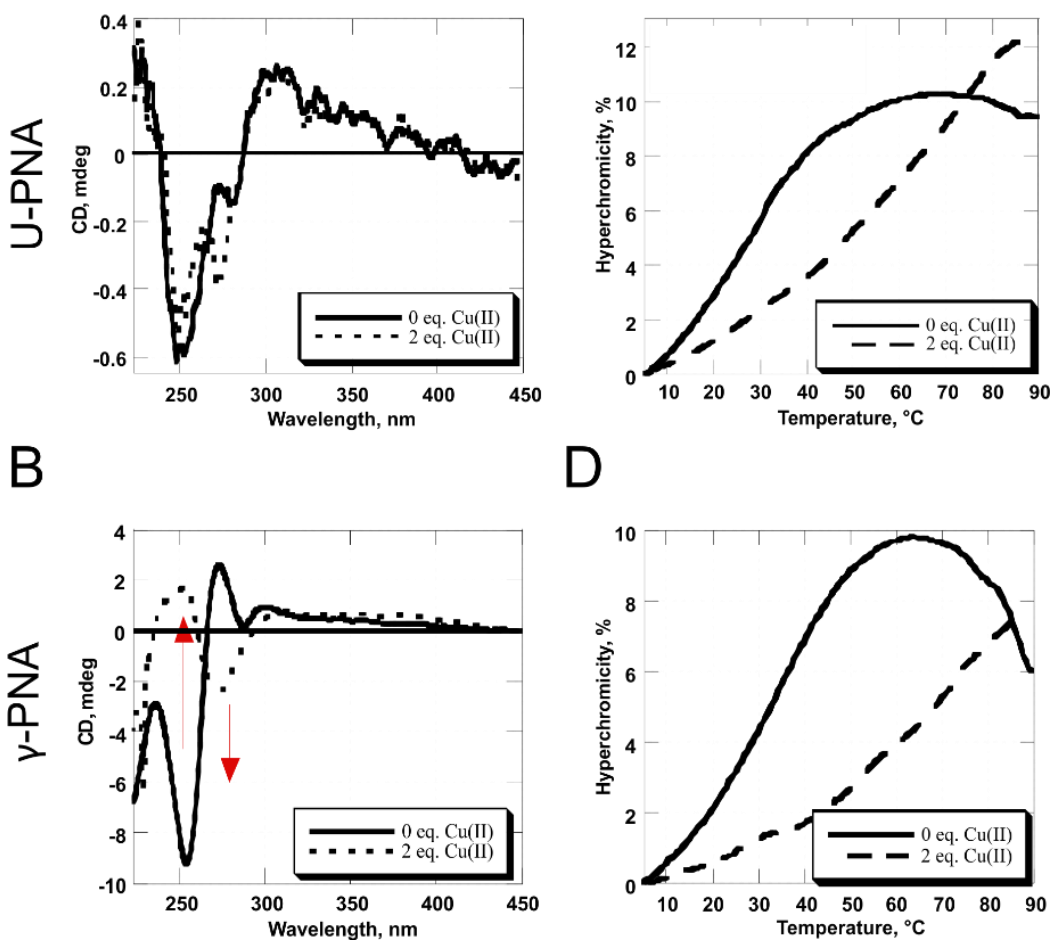

D

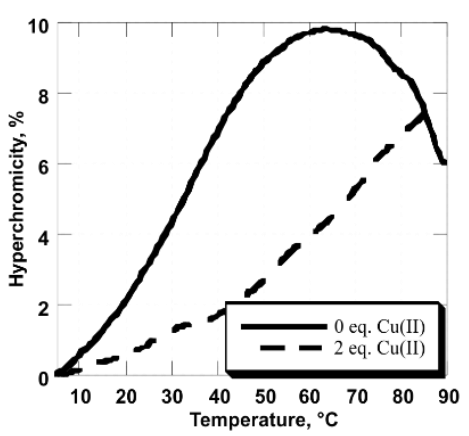

Figure S6. Circular Dichroism data for U-PNA (A) and $\gamma$-PNA (B). Thermal UV/Vis curves for U-PNA (C) and $\gamma$-PNA (D). Each experiment was performed on the PNA in the presence and absence of 2 equivalents of $\mathrm{Cu}^{2+}$ as indicated.

CD spectra for U-PNA and $\gamma$-PNA have been measured in the absence and presence of 2 eq of $\mathrm{Cu}^{2+}$ (Fig. S6). The cause of a preferred handedness in the PNA duplexes is the chiral terminal LLys, which is present both PNA duplexes, as well as the $\gamma$-PNA monomers in the $\gamma$-PNA duplex. ${ }^{1}$ The spectra shown in Fig. S6 reveal that in the absence of $\mathrm{Cu}^{2+}$, U-PNA and $\gamma$-PNA adopt a lefthanded structure. ${ }^{2}$ The handedness of the U-PNA duplex is unaffected by the metal coordination to the Q ligands in the PNA (Figure S6A). In contrast, the preferred handedness of the $\gamma$-PNA duplex (Figure S6B) is changed upon metal coordination to the Q ligands.

As well, the thermal stability of the PNA duplexes was studied by monitoring the absorbance at $\lambda_{\max }=260 \mathrm{~nm}$ as a function of temperature, both in the absence and presence of $\mathrm{Cu}^{2+}$ ions (Fig. 
S6C and D). The melting temperature of the non-modified PNA duplex in $10 \mathrm{mM}$ solutions in $\mathrm{pH}$ 7.0 phosphate buffer is $67^{\circ} \mathrm{C}$. The U-PNA melting curves without added $\mathrm{Cu}^{2+}$, shown in Fig. S6C as the solid line. showed an increase of hyperchromicity that allows one to establish $\sim 26^{\circ} \mathrm{C}$ as an upper limit for the melting temperature of the duplex, a result that is in agreement with the low mismatch tolerance of PNA. ${ }^{3}$ Upon addition of two equivalents of $\mathrm{Cu}^{2+}$, shown as the dashed line in Fig. S6C, the melting temperature increased to $\sim 65^{\circ} \mathrm{C}$. Incorporation of $\gamma$-modified monomers in $\gamma$-PNA did not cause a significant increase in the thermal stability of the duplex relative to UPNA, shown in Fig. S6D as the solid line, with an upper limit of $\sim 31 \mathrm{C}$. Again, with two equivalents of $\mathrm{Cu}^{2+}$ added, shown in Fig. S6D as the dashed line, the melting temperature increased to $\sim 65-$ $70^{\circ} \mathrm{C}$. In both PNA duplexes, the $\mathrm{Cu}^{2+}$ has a stabilizing effect of around $\sim 30-40^{\circ} \mathrm{C}$. 

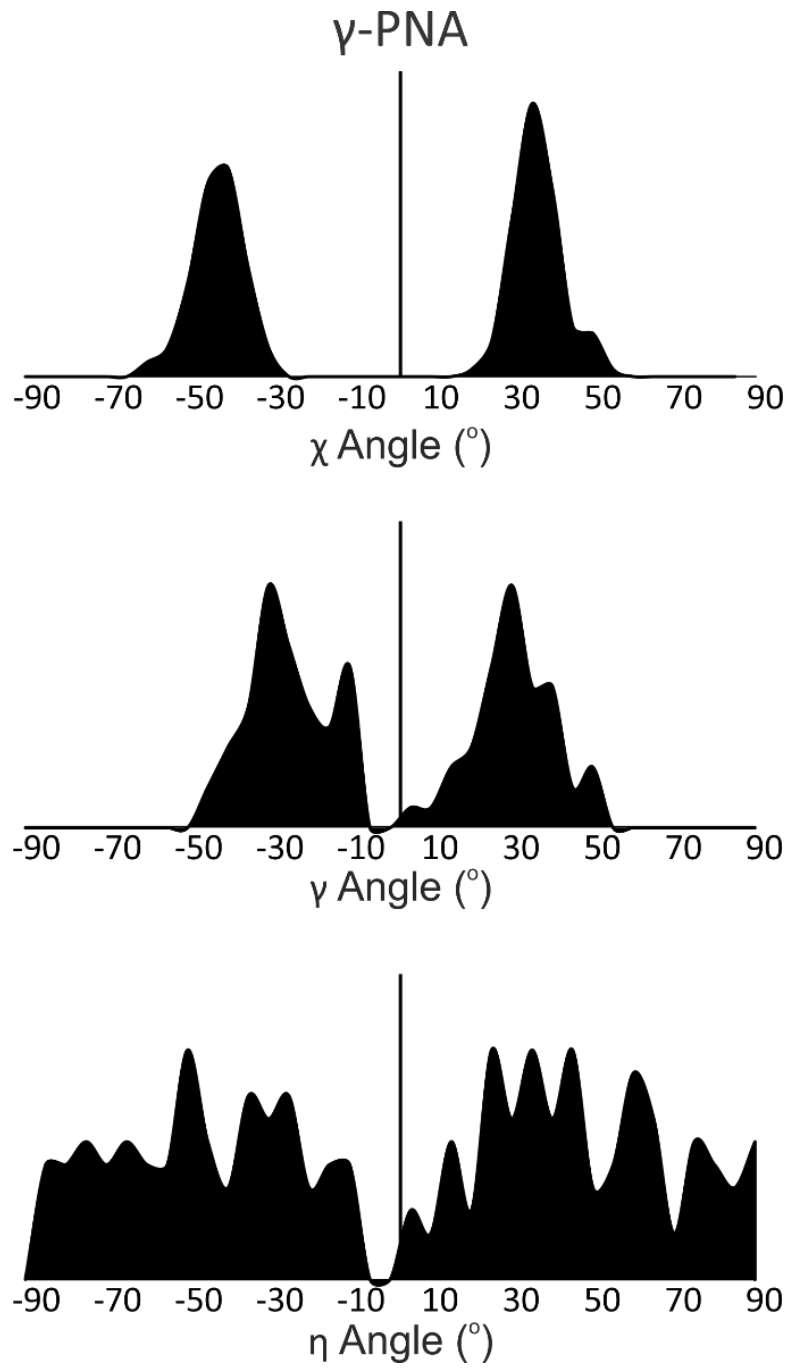

Figure S7. Probability distributions for each of the three orientational angles defined in Figure 5B (Main Text), calculated for $\gamma$-PNA via ORCA. ${ }^{4-5}$ Notably, the average angles and standard deviations are similar in magnitude to those determined for U-PNA. 

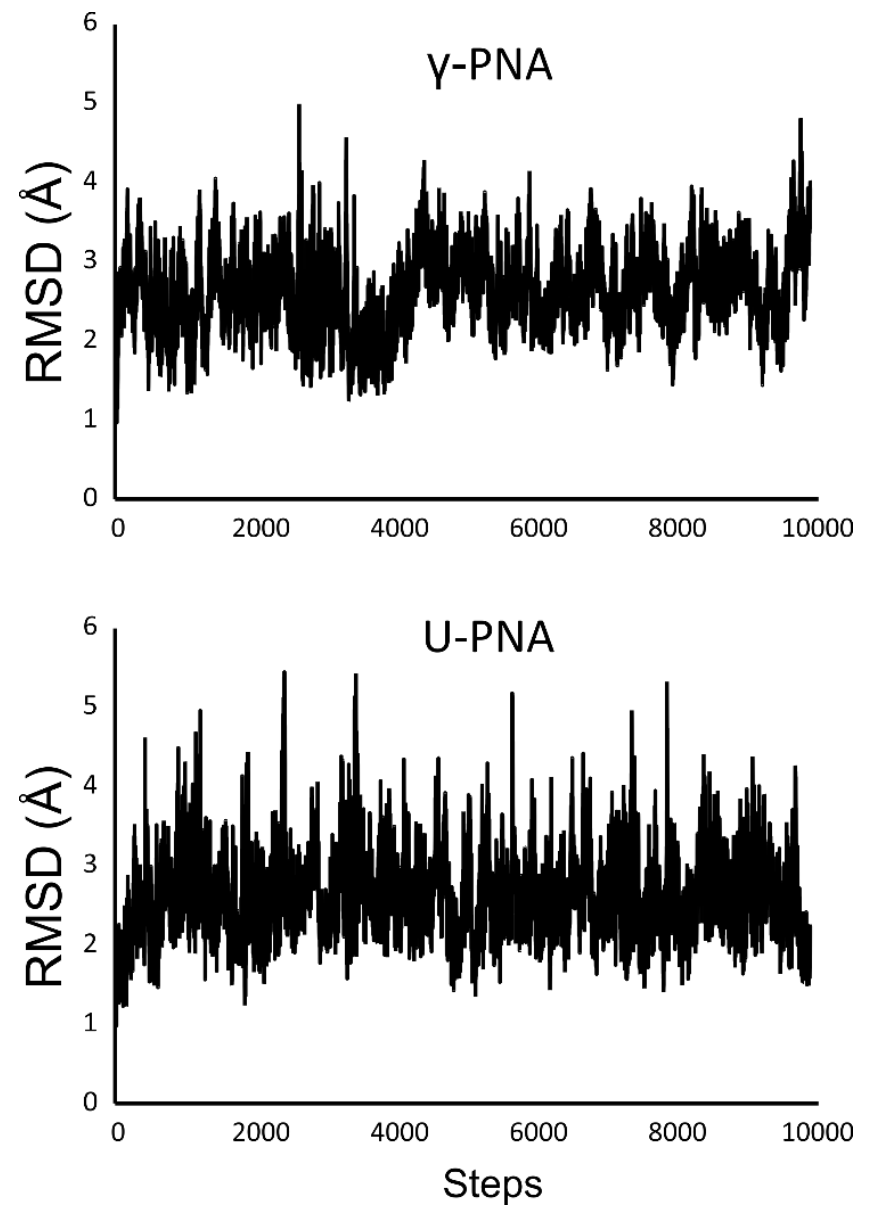

Figure S8. RMSD plots for U-PNA (top) and $\gamma$-PNA (bottom) for each point in the MD trajectory. RMSDs calculated in VMD. ${ }^{6}$ 


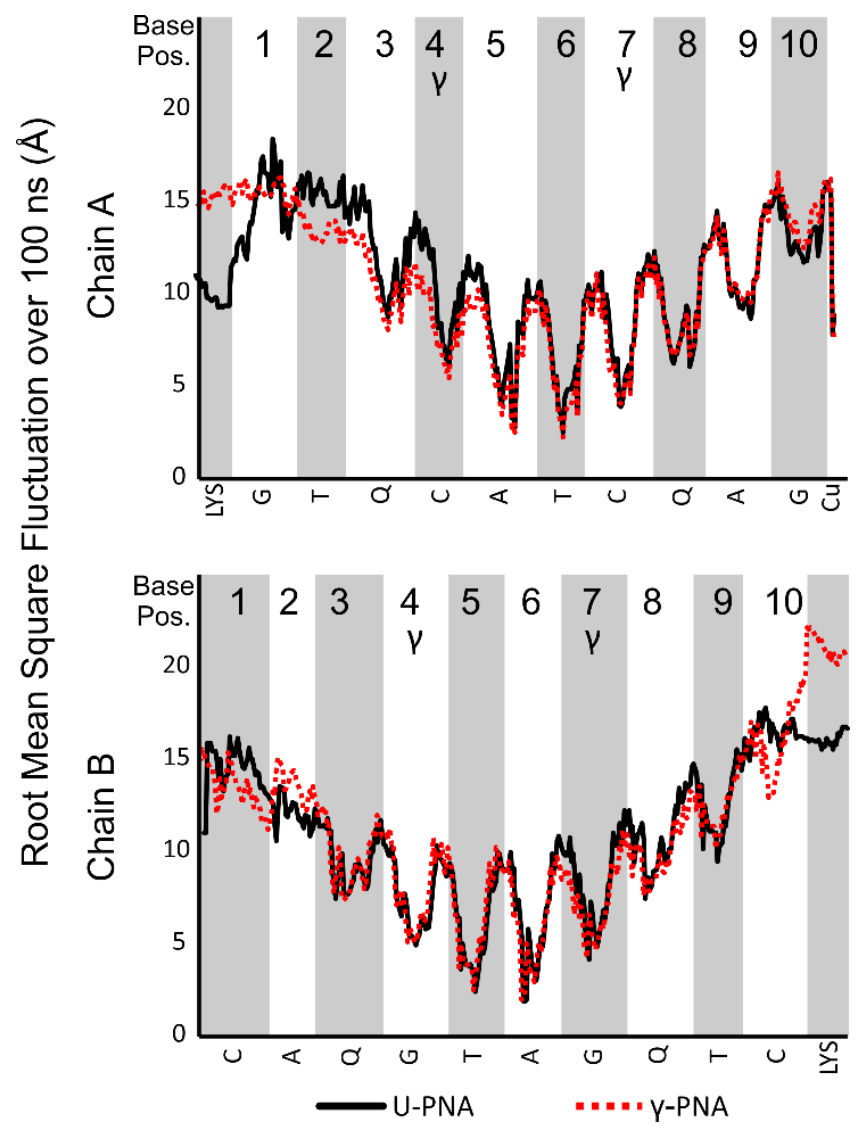

Figure S9. RMSF (root mean square fluctuation) of U-PNA (black line) compared with $\gamma$-PNA (dashed red line) for each nucleobase on both chains of the PNA duplexes. RMSFs calculated in VMD. ${ }^{6}$ 
APN Residue Topology

$0 \quad 0 \quad 2$

This is a remark line

APN.res

APN INT 0

CORRECT OMIT DU BEG

0.0000

$\begin{array}{llllrrrrrrr}1 & \text { DUMM } & \text { DU } & \text { M } & 0 & -1 & -2 & 0.000 & .0 & .0 & .00000 \\ 2 & \text { DUMM } & \text { DU } & \text { M } & 1 & 0 & -1 & 1.449 & .0 & .0 & .00000 \\ 3 & \text { DUMM } & \text { DU } & \text { M } & 2 & 1 & 0 & 1.523 & 111.21 & .0 & .00000\end{array}$

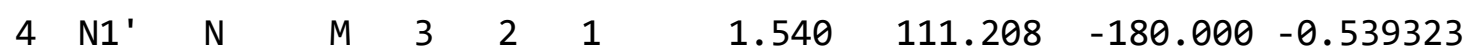

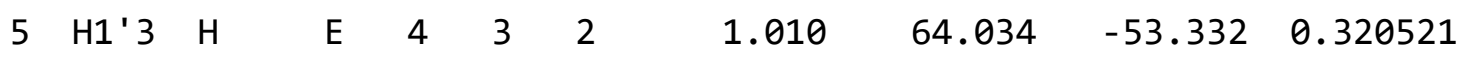

$\begin{array}{lllllllllll}6 & \mathrm{C}^{\prime} & \text { CT } & \text { M } & 4 & 3 & 2 & 1.456 & 114.984 & -163.583 & -0.081334\end{array}$

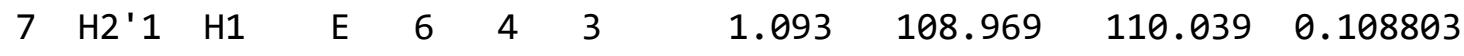

$\begin{array}{llllllllll}8 & H & H & \prime\end{array}$

$\begin{array}{lllllllllll}9 \text { C3 }^{\prime} & \text { CT } & \text { M } & 6 & 4 & 3 & 1.537 & 114.210 & -10.329 & 0.105817\end{array}$

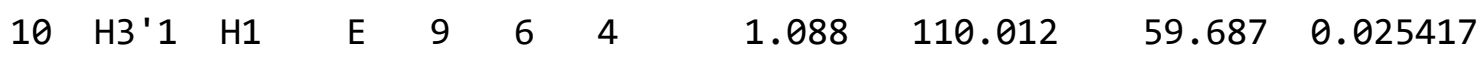

$\begin{array}{llllllllll}11 & \mathrm{H} 3 & & \end{array}$

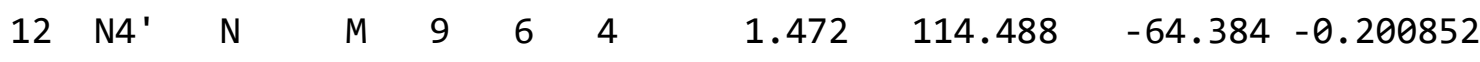

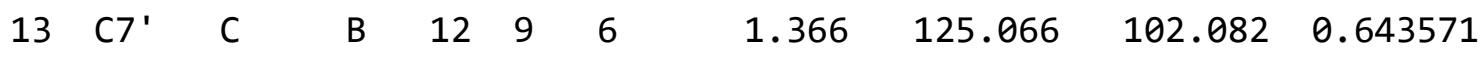

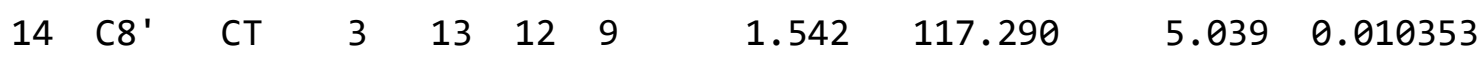

$\begin{array}{lllllllllll}15 & N 9 & N^{*} & \mathrm{~S} & 14 & 13 & 12 & 1.447 & 111.501 & 178.122 & -0.268528\end{array}$

$\begin{array}{lllllllllll}16 & \mathrm{C} 4 & \mathrm{CB} & \mathrm{S} & 15 & 14 & 13 & 1.377 & 126.474 & -89.061 & 0.529267\end{array}$

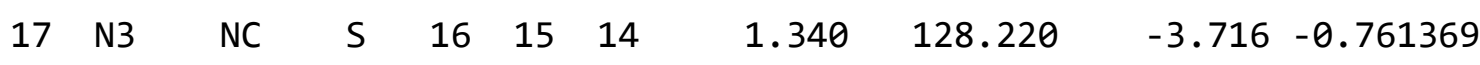

$\begin{array}{lllllllllll}18 & \text { C2 } & \text { CQ } & \text { B } & 17 & 16 & 15 & 1.334 & 111.703 & -179.215 & 0.576680\end{array}$ 


\begin{tabular}{|c|c|c|c|c|c|c|c|c|c|c|}
\hline 19 & $\mathrm{~N} 1$ & NC & $S$ & 18 & 17 & 16 & 1.341 & 128.406 & 0.104 & -0.805542 \\
\hline 20 & $\mathrm{C} 6$ & $C A$ & B & 19 & 18 & 17 & 1.349 & 118.831 & -0.024 & 0.831439 \\
\hline 21 & $\mathrm{C} 5$ & $C B$ & $S$ & 20 & 19 & 18 & 1.410 & 118.263 & -0.109 & -0.049680 \\
\hline 22 & N7 & NB & $S$ & 21 & 20 & 19 & 1.384 & 132.840 & 179.781 & -0.579891 \\
\hline 23 & $\mathrm{C} 8$ & CK & $S$ & 22 & 21 & 20 & 1.312 & 104.138 & -179.673 & 0.236328 \\
\hline 24 & $\mathrm{H} 8$ & $\mathrm{H} 5$ & $E$ & 23 & 22 & 21 & 1.080 & 125.292 & -179.953 & 0.143134 \\
\hline 25 & N6 & N2 & B & 20 & 19 & 18 & 1.348 & 118.957 & 179.923 & -1.016263 \\
\hline 26 & HN61 & $\mathrm{H}$ & $E$ & 25 & 20 & 19 & 1.006 & 120.872 & -179.693 & 0.444669 \\
\hline 27 & HN62 & $\mathrm{H}$ & $E$ & 25 & 20 & 19 & 1.007 & 119.838 & -0.274 & 0.444669 \\
\hline 28 & $\mathrm{H} 2$ & $\mathrm{H} 5$ & $E$ & 18 & 17 & 16 & 1.086 & 116.121 & 179.978 & 0.069967 \\
\hline 29 & H8 ' 1 & $\mathrm{H} 1$ & $E$ & 14 & 13 & 12 & 1.089 & 110.667 & -60.639 & 0.062323 \\
\hline 30 & H8 ' 2 & $\mathrm{H} 1$ & $E$ & 14 & 13 & 12 & 1.092 & 110.107 & 58.572 & 0.062323 \\
\hline 31 & $07^{\prime}$ & 0 & $E$ & 13 & 12 & 9 & 1.226 & 122.384 & -176.336 & -0.549420 \\
\hline 32 & $\mathrm{C} 5^{\prime}$ & $\mathrm{CT}$ & M & 12 & 9 & 6 & 1.462 & 116.963 & -72.296 & -0.511949 \\
\hline 33 & H5 ' 1 & $\mathrm{H} 1$ & $E$ & 32 & 12 & 9 & 1.089 & 109.136 & 147.702 & 0.213327 \\
\hline 34 & H5 ' 2 & $\mathrm{H} 1$ & $E$ & 32 & 12 & 9 & 1.090 & 109.435 & 30.550 & 0.213327 \\
\hline 35 & $C^{\prime}$ & $C$ & M & 32 & 12 & 9 & 1.534 & 116.793 & -90.964 & 0.821407 \\
\hline & $01^{\prime}$ & 0 & $\mathrm{E}$ & 35 & 32 & 12 & 1.233 & 118.843 & 175.443 & -0.633413 \\
\hline
\end{tabular}

LOOP

C8 N9

C5 C4

IMPROPER

$$
\begin{array}{llll}
-\mathrm{M} & \mathrm{C} 2^{\prime} & \mathrm{N} 1^{\prime} & \mathrm{H} 1^{\prime} 3 \\
\mathrm{C}^{\prime} & \mathrm{C} 5^{\prime} & \mathrm{N} 4^{\prime} & \mathrm{C} 3^{\prime} \\
\mathrm{C}^{\prime} & \mathrm{N} 44^{\prime} & \mathrm{C} 7^{\prime} & \mathrm{O} 7^{\prime}
\end{array}
$$




$\begin{array}{llll}\text { C4 } & \text { C8 } & \text { N9 } & \text { C8 }^{\prime} \\ \text { C5 } & \text { N9 } & \text { C4 } & \text { N3 } \\ \text { H2 } & \text { N3 } & \text { C2 } & \text { N1 } \\ \text { C5 } & \text { N6 } & \text { C6 } & \text { N1 } \\ \text { C6 } & \text { C4 } & \text { C5 } & \text { N7 } \\ \text { H8 } & \text { N9 } & \text { C8 } & \text { N7 } \\ \text { C6 } & \text { HN61 } & \text { N6 } & \text { HN62 } \\ \text { C5 ' } & +M & C^{\prime} & \text { 01' } \\ & & & \\ \text { DONE } & & & \\ \text { STOP } & & & \end{array}$


APR Residue Topology

$0 \quad 0 \quad 2$

This is a remark line

APR.res

APR INT 0

CORRECT OMIT DU BEG

0.0000

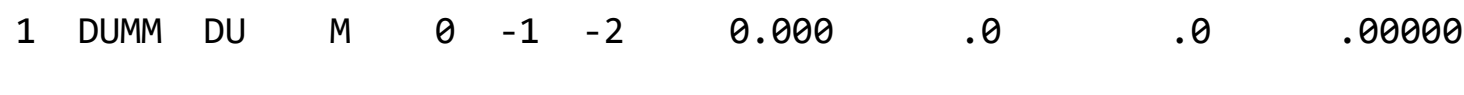

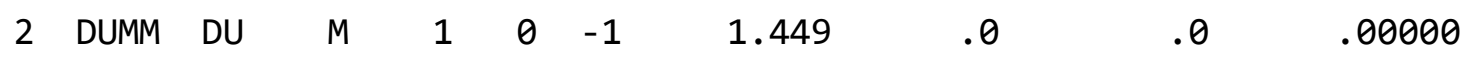

$\begin{array}{llllllllll}3 & \text { DUMM DU } & \text { M } & 2 & 1 & 0 & 1.523 & 111.21 & .0 & .00000\end{array}$

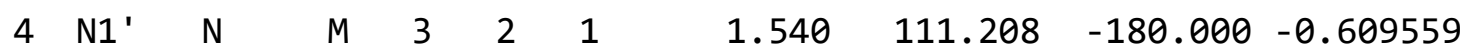

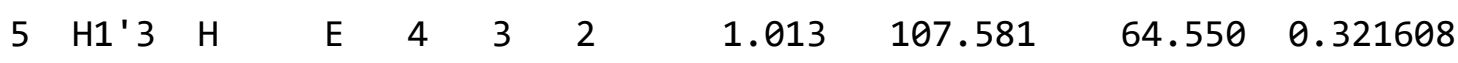

$\begin{array}{lllllllllll}6 & \mathrm{C}^{\prime} & \text { CT } & \text { M } & 4 & 3 & 2 & 1.457 & 65.074 & 173.612 & 0.308140\end{array}$

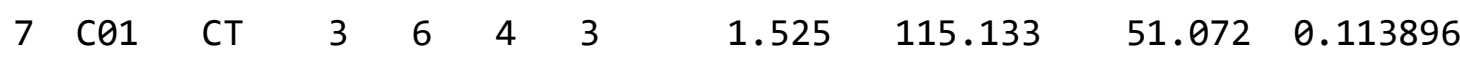

$\begin{array}{lllllllllll}8 & 001 & \mathrm{OH} & \mathrm{S} & 7 & 6 & 4 & 1.408 & 108.221 & -52.809 & -0.685026\end{array}$

$\begin{array}{lllllllllll}9 & \mathrm{H} 01 & \mathrm{HO} & \mathrm{E} & 8 & 7 & 6 & 0.964 & 108.207 & -175.155 & 0.453388\end{array}$

$\begin{array}{lllllllllll}10 & \mathrm{H} 02 & \mathrm{H} 1 & \mathrm{E} & 7 & 6 & 4 & 1.100 & 106.934 & -173.393 & 0.045115\end{array}$

$\begin{array}{lllllllllll}11 & \mathrm{H} 03 & \mathrm{H} 1 & \mathrm{E} & 7 & 6 & 4 & 1.097 & 111.121 & 69.722 & 0.045115\end{array}$

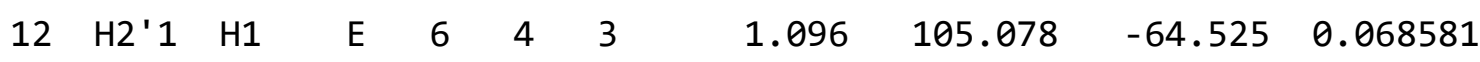

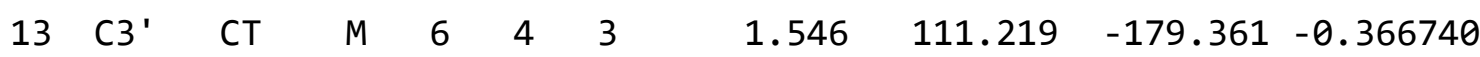

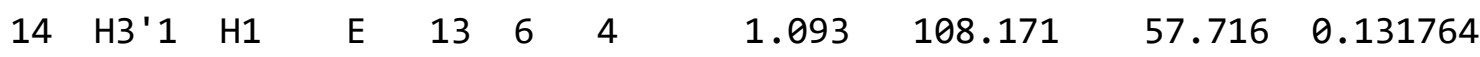

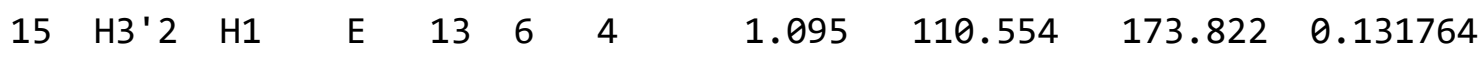

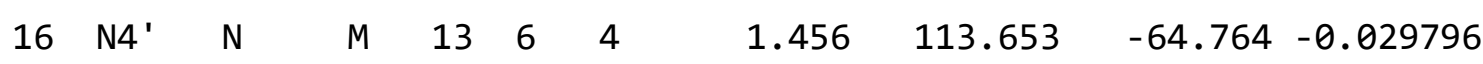

$\begin{array}{lllllllllll}17 & \text { C7 }^{\prime} & \text { C } & \text { B } & 16 & 13 & 6 & 1.367 & 125.243 & 96.999 & 0.564262\end{array}$

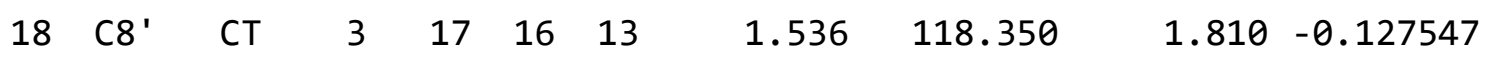




$\begin{array}{lllllllllll}19 & \text { N9 } & \text { N* } & \text { S } & 18 & 17 & 16 & 1.432 & 109.916 & 179.119 & -0.197449 \\ 20 & \text { C4 } & \text { CB } & \text { S } & 19 & 18 & 17 & 1.372 & 125.140 & -76.100 & 0.442288 \\ 21 & \text { N3 } & \text { NC } & \text { S } & 20 & 19 & 18 & 1.338 & 127.854 & -7.789 & -0.705080 \\ 22 & \text { C2 } & \text { CQ } & \text { B } & 21 & 20 & 19 & 1.330 & 110.906 & -179.931 & 0.572076 \\ 23 & \text { N1 } & \text { NC } & \text { S } & 22 & 21 & 20 & 1.340 & 128.961 & 0.004 & -0.797582 \\ 24 & \text { C6 } & \text { CA } & \text { B } & 23 & 22 & 21 & 1.341 & 118.276 & 0.202 & 0.787464 \\ 25 & \text { C5 } & \text { CB } & \text { S } & 24 & 23 & 22 & 1.406 & 118.923 & -0.477 & -0.006019 \\ 26 & \text { N7 } & \text { NB } & \text { S } & 25 & 24 & 23 & 1.383 & 132.919 & -178.075 & -0.600027 \\ 27 & \text { C8 } & \text { CK } & \text { S } & 26 & 25 & 24 & 1.306 & 103.727 & 178.765 & 0.260047 \\ 28 & \text { H8 } & \text { H5 } & \text { E } & 27 & 26 & 25 & 1.084 & 126.088 & -179.012 & 0.156146 \\ 29 & \text { N6 } & \text { N2 } & \text { B } & 24 & 23 & 22 & 1.352 & 118.929 & 178.651 & -0.983654 \\ 30 & \text { HN61 } & \text { H } & \text { E } & 29 & 24 & 23 & 1.008 & 118.897 & 168.908 & 0.431745 \\ 31 & \text { HN62 } & \text { H } & \text { E } & 29 & 24 & 23 & 1.007 & 117.983 & 11.553 & 0.431745 \\ 32 & \text { H2 } & \text { H5 } & \text { E } & 22 & 21 & 20 & 1.088 & 115.961 & -179.770 & 0.060641 \\ 33 & \text { H8'1 } & \text { H1 } & \text { E } & 18 & 17 & 16 & 1.089 & 112.468 & -59.626 & 0.112653 \\ 34 & \text { H8'2 } & \text { H1 } & \text { E } & 18 & 17 & 16 & 1.096 & 108.542 & 61.077 & 0.112653 \\ 35 & \text { 07' } & \text { O } & \text { E } & 17 & 16 & 13 & 1.223 & 121.423 & 178.788 & -0.569623 \\ 36 & \text { C5' } & \text { CT } & \text { M } & 16 & 13 & 6 & 1.466 & 117.239 & -96.885 & -0.171169 \\ 37 & \text { H04 } & \text { H1 } & \text { E } & 36 & 16 & 13 & 1.089 & 109.491 & 33.393 & 0.066876 \\ 38 & \text { H5'1 } & \text { H1 } & \text { E } & 36 & 16 & 13 & 1.091 & 108.744 & 153.328 & 0.066876 \\ 40 & \text { 01' } & \text { O } & \text { E } & 39 & 36 & 16 & 1.224 & 120.637 & 96.456 & -0.609630\end{array}$

LOOP

C8 N9

C5 C4 


\section{IMPROPER}

\begin{tabular}{|c|c|c|c|}
\hline$-M$ & $C 2^{\prime}$ & N1' & $\mathrm{H} 1{ }^{\prime} 3$ \\
\hline C7' & $C 3^{\prime}$ & N4 ' & $C 5^{\prime}$ \\
\hline C8' & N4' & $C 7^{\prime}$ & '07' \\
\hline C4 & C8 & N9 & C8' \\
\hline C5 & N9 & C4 & N3 \\
\hline $\mathrm{H} 2$ & N3 & $\mathrm{C} 2$ & N1 \\
\hline C5 & N6 & C6 & $\mathrm{N} 1$ \\
\hline C6 & C4 & C5 & N7 \\
\hline H8 & N9 & C8 & N7 \\
\hline C6 & HN61 & N6 & HN62 \\
\hline C5 ${ }^{\prime}$ & $+M$ & $C^{\prime}$ & $01^{\prime}$ \\
\hline
\end{tabular}

DONE

STOP 
CPN Residue Topology

$\begin{array}{lll}0 & 0 & 2\end{array}$

This is a remark line

CPN.res

CPN INT 0

CORRECT OMIT DU BEG

0.0000

1 DUMM DU M 0

0.000

.0

.00000

2 DUMM DU M 11000

1.449

.0

.00000

3 DUMM DU M $2 \quad 1 \quad 0$

$1.523 \quad 111.21$

0

.00000

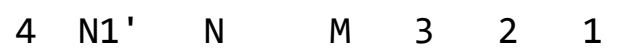

$1.540 \quad 111.208 \quad-180.000 \quad-0.532182$

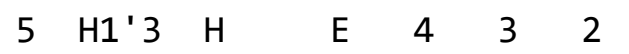

$\begin{array}{llll}1.009 & 58.318 & 121.525 & 0.315311\end{array}$

$\begin{array}{lllllll}6 & \mathrm{C} 2 & \\ \end{array}$

$\begin{array}{llll}1.456 & 127.294 & 18.842 & -0.030986\end{array}$

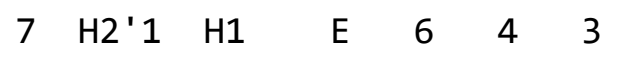

$\begin{array}{llll}1.093 & 108.934 & 108.670 & 0.091559\end{array}$

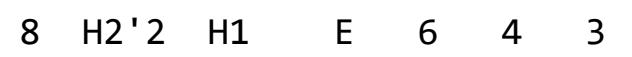

$\begin{array}{llll}1.089 & 107.485 & -134.859 & 0.091559\end{array}$

$\begin{array}{lllllll}9 & \mathrm{C}^{\prime} & \mathrm{CT} & \mathrm{M} & 6 & 4 & 3\end{array}$

$\begin{array}{llll}1.538 & 114.065 & -11.643 & 0.071468\end{array}$

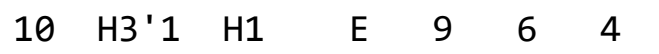

$\begin{array}{llll}1.088 & 109.873 & 58.649 & 0.034484\end{array}$

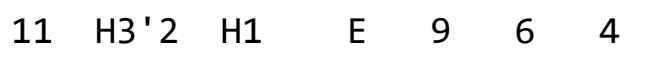

$\begin{array}{llll}1.093 & 108.091 & 174.462 & 0.034484\end{array}$

$\begin{array}{lllllll}12 & N 4^{\prime} & N & M & 9 & 6 & 4\end{array}$

$1.471 \quad 114.401 \quad-65.063-0.144670$

$\begin{array}{lllllll}13 & \mathrm{C}^{\prime} & \mathrm{C} & \mathrm{B} & 12 & 9 & 6\end{array}$

$\begin{array}{llll}1.367 & 125.075 & 100.753 & 0.623361\end{array}$

$\begin{array}{lllllll}14 & \mathrm{C}^{\prime} & \mathrm{CT} & 3 & 13 & 12 & 9\end{array}$

$\begin{array}{llll}1.540 & 117.544 & 5.454 & -0.060343\end{array}$

$\begin{array}{lllllll}15 & \mathrm{~N} 1 & \mathrm{~N} * & \mathrm{~S} & 14 & 13 & 12\end{array}$

$\begin{array}{llll}1.455 & 111.258 & 171.908 & -0.291067\end{array}$

$\begin{array}{lllllll}16 & \text { C2 } & \text { C } & \text { B } & 15 & 14 & 13\end{array}$

$\begin{array}{llll}1.419 & 118.384 & -83.350 & 0.917682\end{array}$

$\begin{array}{lllllll}17 & 02 & 0 & \text { E } & 16 & 15 & 14\end{array}$

$\begin{array}{llll}1.235 & 118.522 & -2.223 & -0.649242\end{array}$

$\begin{array}{lllllll}18 & \text { N3 } & \text { NC } & \text { S } & 16 & 15 & 14\end{array}$

$\begin{array}{llll}1.358 & 118.088 & 178.162 & -0.877686\end{array}$ 


\begin{tabular}{|c|c|c|c|c|c|c|c|c|c|c|}
\hline 19 & $\mathrm{C} 4$ & $\mathrm{CA}$ & B & 18 & 16 & 15 & 1.330 & 120.632 & -1.644 & 1.123327 \\
\hline$a$ & N4 & N2 & B & 19 & 18 & 16 & 1.349 & 117.583 & -179.946 & -1.159356 \\
\hline 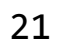 & HN41 & $\mathrm{H}$ & $E$ & 20 & 19 & 18 & 1.007 & 121.606 & 177.896 & 0.477501 \\
\hline 22 & HN42 & $\mathrm{H}$ & $E$ & 20 & 19 & 18 & 1.008 & 119.537 & 1.749 & 0.477501 \\
\hline 23 & $\mathrm{C} 5$ & $\mathrm{CM}$ & B & 19 & 18 & 16 & 1.432 & 122.550 & 0.304 & -0.722986 \\
\hline 4 & $\mathrm{C} 6$ & $\mathrm{CM}$ & $S$ & 23 & 19 & 18 & 1.355 & 116.574 & 0.309 & 0.145768 \\
\hline 5 & $\mathrm{H} 6$ & $\mathrm{H} 4$ & $E$ & 24 & 23 & 19 & 1.082 & 122.347 & -179.859 & 0.183478 \\
\hline 6 & $\mathrm{H} 5$ & $\mathrm{HA}$ & $E$ & 23 & 19 & 18 & 1.080 & 122.168 & -179.924 & 0.236195 \\
\hline 27 & $\mathrm{H} 8$ ' 1 & $\mathrm{H} 1$ & $E$ & 14 & 13 & 12 & 1.088 & 110.437 & 52.132 & 0.064456 \\
\hline 28 & H8 ' 2 & $\mathrm{H} 1$ & $E$ & 14 & 13 & 12 & 1.089 & 109.946 & -67.531 & 0.064456 \\
\hline 29 & $07^{\prime}$ & 0 & $E$ & 13 & 12 & 9 & 1.227 & 122.033 & -175.283 & -0.551892 \\
\hline 30 & $\mathrm{C} 5^{\prime}$ & $\mathrm{CT}$ & M & 12 & 9 & 6 & 1.462 & 117.004 & -72.449 & -0.585258 \\
\hline 31 & H5 ' 1 & $\mathrm{H} 1$ & E & 30 & 12 & 9 & 1.089 & 109.471 & 142.792 & 0.229027 \\
\hline 32 & H5 ' 2 & $\mathrm{H} 1$ & $E$ & 30 & 12 & 9 & 1.091 & 109.197 & 25.476 & 0.229027 \\
\hline 3 & $C^{\prime}$ & $\mathrm{C}$ & M & 30 & 12 & 9 & 1.533 & 116.735 & -95.645 & 0.833568 \\
\hline & $01^{\prime}$ & 0 & $E$ & 33 & 30 & 12 & 1.233 & 118.892 & 177.859 & -0.638544 \\
\hline
\end{tabular}

LOOP

C6 N1

\section{IMPROPER}

$\begin{array}{cccc}-\mathrm{M} & \mathrm{C} 2^{\prime} & \mathrm{N} 1^{\prime} & \mathrm{H} 1^{\prime} 3 \\ \mathrm{C}{ }^{\prime} & \mathrm{C} 5^{\prime} & \mathrm{N} 4{ }^{\prime} & \mathrm{C} 3^{\prime} \\ \mathrm{C} 8^{\prime} & \mathrm{N} 4{ }^{\prime} & \mathrm{C} 7^{\prime} & \mathrm{O} 7^{\prime} \\ \mathrm{C} 2 & \mathrm{C} 6 & \mathrm{~N} 1 & \mathrm{C} 8^{\prime} \\ \mathrm{N} 1 & \mathrm{~N} 3 & \mathrm{C} 2 & \mathrm{O} 2 \\ \mathrm{C} 5 & \mathrm{~N} 4 & \mathrm{C} 4 & \mathrm{~N} 3\end{array}$




$\begin{array}{rrrr}\text { C4 } & \text { HN41 } & \text { N4 } & \text { HN42 } \\ \text { C4 } & \text { C6 } & \text { C5 } & \text { H5 } \\ \text { C5 } & \text { H6 } & \text { C6 } & \text { N1 } \\ \text { C5 }^{\prime} & + \text { M } & \text { C' }^{\prime} & \text { 01 ' }\end{array}$

DONE

STOP 
CPR Residue Topology

$\begin{array}{lll}0 & 0 & 2\end{array}$

This is a remark line

CPR.res

CPR INT 0

CORRECT OMIT DU BEG

0.0000

0.000

.0

.0

.00000

2 DUMM DU M 11000

1.449

.0

.0

.00000

3 DUMM DU $\quad M \quad 2 \quad 1 \quad 0$

$1.523 \quad 111.21$

$.0 \quad .00000$

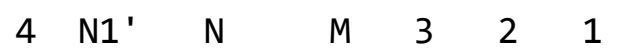

$\begin{array}{llll}1.540 & 111.208 & -180.000 & -0.614029\end{array}$

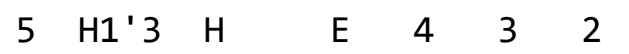

$\begin{array}{llll}1.013 & 68.083 & -52.264 & 0.310046\end{array}$

$\begin{array}{lllllll}6 & \mathrm{C} 01 & \mathrm{CT} & \mathrm{M} & 4 & 3 & 2\end{array}$

$\begin{array}{llll}1.447 & 101.480 & -167.414 & 0.607158\end{array}$

$\begin{array}{lllllll}7 & \mathrm{C} 02 & \mathrm{CT} & 3 & 6 & 4 & 3\end{array}$

$\begin{array}{llll}1.524 & 110.675 & 119.397 & 0.017565\end{array}$

$\begin{array}{lllllll}8 & 001 & \mathrm{OH} & \mathrm{S} & 7 & 6 & 4\end{array}$

$\begin{array}{llll}1.407 & 107.991 & 62.866 & -0.726558\end{array}$

$\begin{array}{lllllll}9 & \mathrm{H} 01 & \mathrm{HO} & \mathrm{E} & 8 & 7 & 6\end{array}$

$\begin{array}{llll}0.963 & 108.381 & -179.203 & 0.474455\end{array}$

$\begin{array}{lllllll}10 & \mathrm{H} 05 & \mathrm{H} 1 & \mathrm{E} & 7 & 6 & 4\end{array}$

$\begin{array}{llll}1.104 & 109.272 & -58.919 & 0.053368\end{array}$

$\begin{array}{lllllll}11 & \mathrm{H} 03 & \mathrm{H} 1 & \mathrm{E} & 7 & 6 & 4\end{array}$

$\begin{array}{llll}1.101 & 108.544 & -175.673 & 0.053368\end{array}$

$\begin{array}{lllllll}12 & \mathrm{H} 02 & \mathrm{H} 1 & \mathrm{E} & 6 & 4 & 3\end{array}$

$\begin{array}{llll}1.093 & 108.064 & -123.448 & 0.024700\end{array}$

$\begin{array}{lllllll}13 & \mathrm{C}^{\prime} & \mathrm{CT} & \mathrm{M} & 6 & 4 & 3\end{array}$

$1.541 \quad 111.221 \quad-4.508-0.618240$

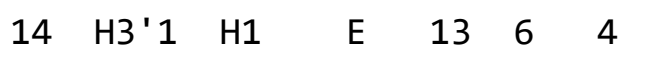

$1.094 \quad 111.510$

$61.756 \quad 0.167824$

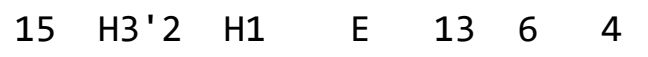

$\begin{array}{llll}1.094 & 109.702 & 178.607 & 0.167824\end{array}$

$\begin{array}{lllllll}16 & N 4^{\prime} & N & M & 13 & 6 & 4\end{array}$

$1.453 \quad 111.945$

$-62.306$

0.060518

$\begin{array}{lllllll}17 & \mathrm{C}^{\prime} & \mathrm{C} & \mathrm{B} & 16 & 13 & 6\end{array}$

$1.366 \quad 125.856$

$90.043 \quad 0.608218$

$\begin{array}{lllllll}18 & \mathrm{C}^{\prime} & \mathrm{CT} & 3 & 17 & 16 & 13\end{array}$

$1.533 \quad 119.842$

$0.718-0.223150$ 


\begin{tabular}{|c|c|c|c|c|c|c|c|c|c|c|}
\hline 19 & $\mathrm{~N} 1$ & $\mathrm{~N}^{*}$ & $S$ & 18 & 17 & 16 & 1.453 & 107.843 & 127.379 & -0.226044 \\
\hline 20 & $\mathrm{C} 2$ & $C$ & B & 19 & 18 & 17 & 1.416 & 116.175 & -69.259 & 0.943586 \\
\hline 21 & 02 & 0 & $E$ & 20 & 19 & 18 & 1.233 & 117.548 & -2.296 & -0.669107 \\
\hline 22 & N3 & $\mathrm{NC}$ & $S$ & 20 & 19 & 18 & 1.354 & 118.222 & 177.687 & -0.860363 \\
\hline 23 & $\mathrm{C} 4$ & CA & B & 22 & 20 & 19 & 1.321 & 119.775 & -4.278 & 1.024680 \\
\hline 24 & N4 & $\mathrm{N} 2$ & B & 23 & 22 & 20 & 1.354 & 116.851 & 178.978 & -1.055869 \\
\hline 25 & HN41 & $\mathrm{H}$ & $E$ & 24 & 23 & 22 & 1.006 & 120.990 & 167.318 & 0.447865 \\
\hline 26 & HN42 & $\mathrm{H}$ & $E$ & 24 & 23 & 22 & 1.009 & 117.097 & 8.925 & 0.447865 \\
\hline 27 & $\mathrm{C} 5$ & CM & B & 23 & 22 & 20 & 1.431 & 123.483 & -0.231 & -0.694626 \\
\hline 28 & $\mathrm{C} 6$ & $\mathrm{CM}$ & $S$ & 27 & 23 & 22 & 1.355 & 116.185 & 1.893 & 0.148090 \\
\hline 29 & $\mathrm{H} 6$ & $\mathrm{H} 4$ & $E$ & 28 & 27 & 23 & 1.084 & 122.961 & -179.324 & 0.199609 \\
\hline 30 & $\mathrm{H} 5$ & $\mathrm{HA}$ & $E$ & 27 & 23 & 22 & 1.082 & 122.458 & -178.071 & 0.239985 \\
\hline 31 & H8 ' 1 & $\mathrm{H} 1$ & $E$ & 18 & 17 & 16 & 1.093 & 107.971 & -114.288 & 0.127700 \\
\hline 32 & H8 ' 2 & $\mathrm{H} 1$ & $E$ & 18 & 17 & 16 & 1.086 & 114.609 & 6.784 & 0.127700 \\
\hline 33 & $07^{\prime}$ & 0 & $E$ & 17 & 16 & 13 & 1.221 & 122.463 & 179.316 & -0.598532 \\
\hline 34 & $\mathrm{C5}{ }^{\prime}$ & CT & M & 16 & 13 & 6 & 1.453 & 116.156 & -96.102 & -0.496976 \\
\hline 35 & H5 ' 1 & $\mathrm{H} 1$ & $E$ & 34 & 16 & 13 & 1.091 & 108.514 & 163.925 & 0.166311 \\
\hline 36 & H5 ' 2 & $\mathrm{H} 1$ & $E$ & 34 & 16 & 13 & 1.095 & 110.227 & 45.543 & 0.166311 \\
\hline 37 & $C^{\prime}$ & $C$ & M & 34 & 16 & 13 & 1.535 & 114.447 & -75.537 & 0.843011 \\
\hline 38 & $01^{\prime}$ & 0 & $E$ & 37 & 34 & 16 & 1.223 & 119.484 & 166.080 & -0.644263 \\
\hline
\end{tabular}

LOOP

C6 N1

IMPROPER

$\begin{array}{cccc}-\mathrm{M} & \mathrm{CO} 1 & \mathrm{~N} 1^{\prime} & \mathrm{H} 1^{\prime} 3 \\ \mathrm{C}^{\prime} & \mathrm{C} 3^{\prime} & \mathrm{N} 4^{\prime} & \mathrm{C} 5^{\prime}\end{array}$ 


$\begin{array}{lrrr}\mathrm{C} 8{ }^{\prime} & \mathrm{N}^{\prime} & \mathrm{C}^{\prime} & \mathrm{O}^{\prime} \\ \mathrm{C} 2 & \mathrm{C} 6 & \mathrm{~N} 1 & \mathrm{C} 8^{\prime} \\ \mathrm{N} 1 & \mathrm{~N} 3 & \mathrm{C} 2 & \mathrm{O} 2 \\ \mathrm{C} 5 & \mathrm{~N} 4 & \mathrm{C} 4 & \mathrm{~N} 3 \\ \mathrm{C} 4 & \mathrm{HN} 41 & \mathrm{~N} 4 & \mathrm{HN} 42 \\ \mathrm{C} 4 & \mathrm{C} 6 & \mathrm{C} 5 & \mathrm{H} 5 \\ \mathrm{C} 5 & \mathrm{H} 6 & \mathrm{C} 6 & \mathrm{~N} 1 \\ \mathrm{C} 5 & +\mathrm{M} & \mathrm{C}^{\prime} & 01^{\prime}\end{array}$

DONE

STOP 
GPN Residue Topology

$\begin{array}{lll}0 & 0 & 2\end{array}$

This is a remark line

GPN.res

GPN INT 0

CORRECT OMIT DU BEG

0.0000

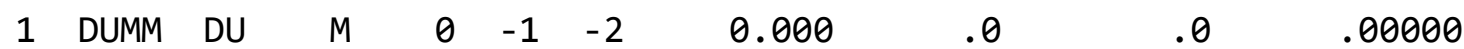

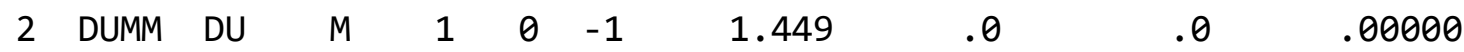

$\begin{array}{llllllllll}3 & \text { DUMM DU } & \text { M } & 2 & 1 & 0 & 1.523 & 111.21 & .0 & .00000\end{array}$

$\begin{array}{lllllllllll}4 & N^{\prime} & N & M & 3 & 2 & 1 & 1.540 & 111.208 & -180.000 & -0.539793\end{array}$

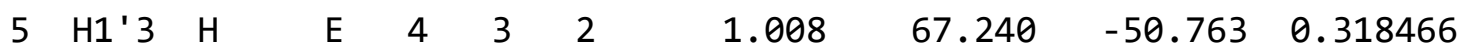

$\begin{array}{lllllllllll}6 & \text { C2 }^{\prime} & \text { CT } & \text { M } & 4 & 3 & 2 & 1.456 & 106.191 & -164.741 & -0.064897\end{array}$

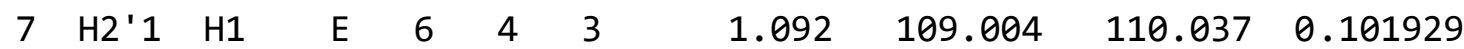

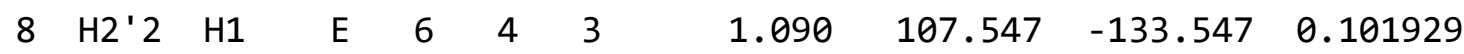

$\begin{array}{lllllllllll}9 & \text { C3 }^{\prime} & \text { CT } & M & 6 & 4 & 3 & 1.538 & 114.124 & -10.304 & 0.086642\end{array}$

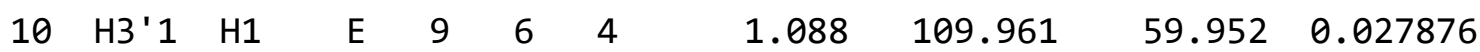

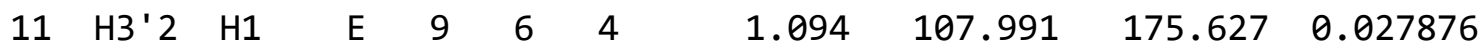

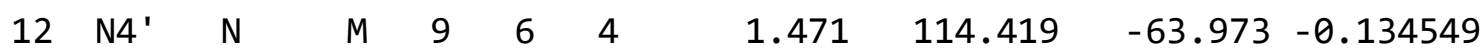

$\begin{array}{lllllllllll}13 & \text { C7 }^{\prime} & \text { C } & \text { B } & 12 & 9 & 6 & 1.367 & 124.960 & 101.645 & 0.599052\end{array}$

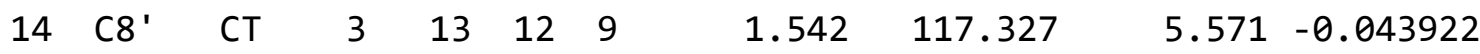

$\begin{array}{lllllllllll}15 & N 9 & N^{*} & \mathrm{~S} & 14 & 13 & 12 & 1.448 & 111.459 & 176.694 & -0.140427\end{array}$

$\begin{array}{lllllllllll}16 & \mathrm{C} 4 & \mathrm{CB} & \mathrm{S} & 15 & 14 & 13 & 1.372 & 126.179 & -86.641 & 0.281806\end{array}$

$\begin{array}{lllllllllll}17 & N 3 & N C & S & 16 & 15 & 14 & 1.349 & 125.975 & -2.852 & -0.695679\end{array}$

$\begin{array}{lllllllllll}18 & \text { C2 } & \text { CA } & \text { B } & 17 & 16 & 15 & 1.317 & 113.259 & -179.242 & 0.929884\end{array}$ 


\begin{tabular}{|c|c|c|c|c|c|c|c|c|c|c|}
\hline 19 & $\mathrm{~N} 1$ & NA & B & 18 & 17 & 16 & 1.374 & 122.820 & -0.421 & -0.737714 \\
\hline 20 & $\mathrm{C} 6$ & $\mathrm{C}$ & $\mathrm{B}$ & 19 & 18 & 17 & 1.418 & 126.147 & 0.232 & 0.649755 \\
\hline 1 & C5 & $C B$ & $S$ & 20 & 19 & 18 & 1.428 & 110.603 & -0.085 & 0.100419 \\
\hline 22 & N7 & NB & $S$ & 21 & 20 & 19 & 1.383 & 130.780 & 179.869 & -0.546293 \\
\hline 23 & $\mathrm{C} 8$ & CK & $S$ & 22 & 21 & 20 & 1.309 & 104.546 & -179.791 & 0.156807 \\
\hline 24 & $\mathrm{H} 8$ & $\mathrm{H} 5$ & $E$ & 23 & 22 & 21 & 1.079 & 125.759 & -179.933 & 0.154868 \\
\hline 5 & 06 & 0 & $E$ & 20 & 19 & 18 & 1.230 & 119.663 & 179.871 & -0.582518 \\
\hline 26 & HN1 & $\mathrm{H}$ & $E$ & 19 & 18 & 17 & 1.014 & 119.224 & 178.898 & 0.408958 \\
\hline 27 & $\mathrm{~N} 2$ & N2 & $\mathrm{B}$ & 18 & 17 & 16 & 1.358 & 119.811 & 177.259 & -1.011526 \\
\hline 28 & HN21 & $\mathrm{H}$ & $E$ & 27 & 18 & 17 & 1.009 & 115.881 & 13.659 & 0.433929 \\
\hline 29 & HN22 & $\mathrm{H}$ & $E$ & 27 & 18 & 17 & 1.009 & 120.001 & 160.600 & 0.433929 \\
\hline 30 & H8 ' 1 & $\mathrm{H} 1$ & $E$ & 14 & 13 & 12 & 1.090 & 110.509 & -62.334 & 0.072149 \\
\hline 31 & H8 ' 2 & $\mathrm{H} 1$ & $E$ & 14 & 13 & 12 & 1.091 & 110.154 & 56.849 & 0.072149 \\
\hline 32 & $07^{\prime}$ & 0 & $E$ & 13 & 12 & 9 & 1.226 & 122.220 & -175.953 & -0.541429 \\
\hline 33 & $\mathrm{C} 5^{\prime}$ & CT & $M$ & 12 & 9 & 6 & 1.462 & 117.044 & -72.105 & -0.542282 \\
\hline 34 & H5 ' 1 & $\mathrm{H} 1$ & $E$ & 33 & 12 & 9 & 1.090 & 109.293 & 144.492 & 0.222701 \\
\hline 35 & H5 ' 2 & $\mathrm{H} 1$ & $E$ & 33 & 12 & 9 & 1.090 & 109.345 & 27.295 & 0.222701 \\
\hline 6 & $C^{\prime}$ & $C$ & $M$ & 33 & 12 & 9 & 1.534 & 116.827 & -94.110 & 0.801149 \\
\hline 37 & $01^{\prime}$ & 0 & $E$ & 36 & 33 & 12 & 1.233 & 118.795 & 177.666 & -0.623947 \\
\hline
\end{tabular}

LOOP

C8 N9

C5 C4

IMPROPER

$\begin{array}{cccc}-M & \mathrm{C} 2^{\prime} & \mathrm{N} 1^{\prime} & \mathrm{H} 1^{\prime} 3 \\ \mathrm{C}^{\prime} & \mathrm{C} 5^{\prime} & \mathrm{N} 44^{\prime} & \mathrm{C} 3^{\prime}\end{array}$ 


\begin{tabular}{|c|c|c|c|}
\hline C8 ' & N4 ' & C7' & 07 \\
\hline C4 & C8 & N9 & C $8^{\prime}$ \\
\hline C5 & N9 & C4 & $\mathrm{N}$ \\
\hline N2 & N1 & $\mathrm{C} 2$ & $\mathrm{~N}^{3}$ \\
\hline C6 & $\mathrm{C} 2$ & N1 & $\mathrm{HN}$ \\
\hline C5 & $\mathrm{N} 1$ & C6 & 0 \\
\hline C6 & C4 & C5 & \\
\hline H8 & N9 & C8 & \\
\hline C2 & HN21 & $\mathrm{N} 2$ & $\mathrm{HN}$ \\
\hline$C 5^{\prime}$ & $+M$ & $C^{\prime}$ & \\
\hline
\end{tabular}

DONE

STOP 
GPR Residue Topology

$\begin{array}{lll}0 & 0 & 2\end{array}$

This is a remark line

GPR.res

GPR INT 0

CORRECT OMIT DU BEG

0.0000

1 DUMM DU $M \quad 0 \begin{array}{lllllll}0 & -1 & -2 & 0.000 & .0 & .0 & .00000\end{array}$

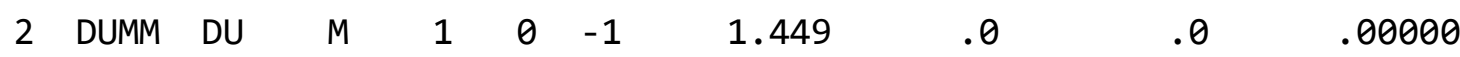

$\begin{array}{llllllllll}3 & \text { DUMM DU } & \text { M } & 2 & 1 & 0 & 1.523 & 111.21 & .0 & .00000\end{array}$

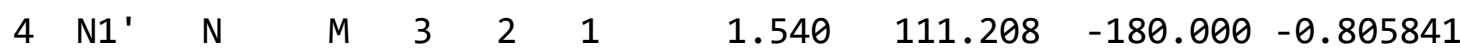

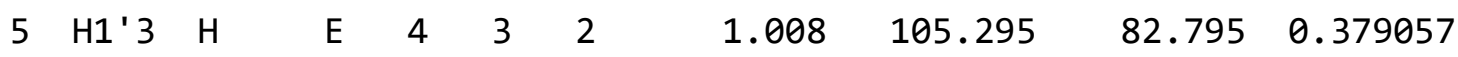

$\begin{array}{lllllllllll}6 & \mathrm{C}^{\prime} & \text { CT } & \text { M } & 4 & 3 & 2 & 1.463 & 128.813 & -130.874 & 0.343211\end{array}$

$\begin{array}{lllllllllll}7 & \mathrm{C} 01 & \mathrm{CT} & 3 & 6 & 4 & 3 & 1.537 & 113.162 & 83.010 & 0.049051\end{array}$

$\begin{array}{lllllllllll}8 & 001 & \mathrm{OH} & \mathrm{S} & 7 & 6 & 4 & 1.413 & 109.940 & 169.049 & -0.671465\end{array}$

$\begin{array}{lllllllllll}9 & \mathrm{H} 01 & \mathrm{HO} & \mathrm{E} & 8 & 7 & 6 & 0.965 & 108.666 & -75.272 & 0.426184\end{array}$

$\begin{array}{lllllllllll}10 & \mathrm{H} 02 & \mathrm{H} 1 & \mathrm{E} & 7 & 6 & 4 & 1.089 & 110.297 & -73.412 & 0.097842\end{array}$

$\begin{array}{lllllllllll}11 & \mathrm{H} 04 & \mathrm{H} 1 & \mathrm{E} & 7 & 6 & 4 & 1.098 & 109.445 & 45.209 & 0.097842\end{array}$

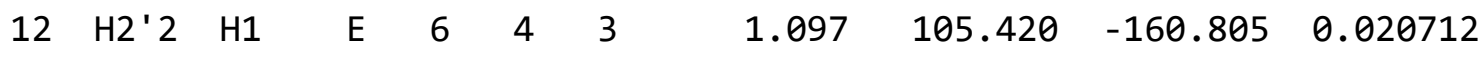

$\begin{array}{lllllllllll}13 & \text { C3 }^{\prime} & \text { CT } & \text { M } & 6 & 4 & 3 & 1.545 & 112.014 & -43.922 & -0.147417\end{array}$

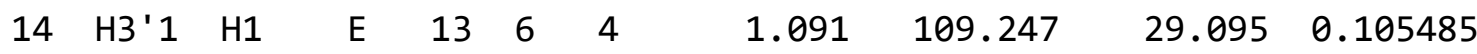

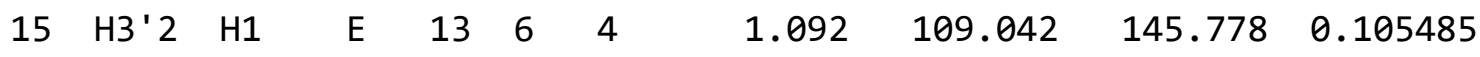

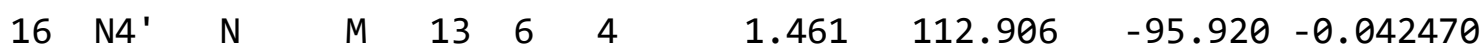

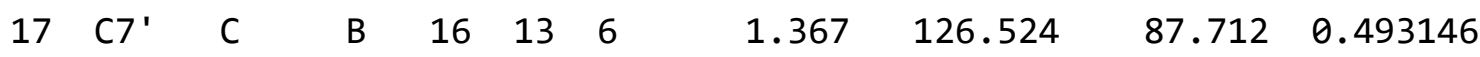

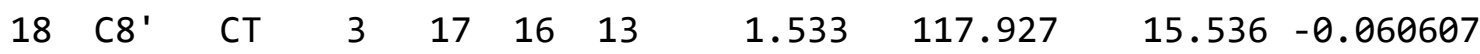




\begin{tabular}{|c|c|c|c|c|c|c|c|c|c|c|}
\hline 19 & N9 & $\mathrm{N}^{*}$ & $\mathrm{~S}$ & 18 & 17 & 16 & 1.451 & 108.716 & 74.194 & -0.058597 \\
\hline 20 & $\mathrm{C} 4$ & $C B$ & $\mathrm{~S}$ & 19 & 18 & 17 & 1.368 & 125.850 & -117.159 & 0.027112 \\
\hline 1 & N3 & NC & $S$ & 20 & 19 & 18 & 1.353 & 124.391 & -4.080 & -0.405937 \\
\hline 22 & $\mathrm{C} 2$ & CA & B & 21 & 20 & 19 & 1.314 & 112.701 & 175.683 & 0.767437 \\
\hline 3 & N1 & NA & B & 22 & 21 & 20 & 1.370 & 122.565 & 3.226 & -0.666592 \\
\hline 4 & $\mathrm{C} 6$ & $C$ & B & 23 & 22 & 21 & 1.433 & 127.034 & -3.548 & 0.596601 \\
\hline 25 & $\mathrm{C} 5$ & $C B$ & $\mathrm{~S}$ & 24 & 23 & 22 & 1.439 & 109.507 & 0.849 & 0.203932 \\
\hline 6 & N7 & NB & $S$ & 25 & 24 & 23 & 1.380 & 130.970 & -176.437 & -0.564220 \\
\hline 27 & $\mathrm{C} 8$ & CK & $S$ & 26 & 25 & 24 & 1.305 & 104.531 & 178.284 & 0.164287 \\
\hline 8 & $\mathrm{H} 8$ & $\mathrm{H} 5$ & $E$ & 27 & 26 & 25 & 1.081 & 126.121 & -179.276 & 0.166072 \\
\hline 9 & 06 & 0 & $\mathrm{E}$ & 24 & 23 & 22 & 1.212 & 119.308 & -178.485 & -0.557575 \\
\hline 0 & HN1 & $\mathrm{H}$ & $E$ & 23 & 22 & 21 & 1.012 & 119.853 & 177.604 & 0.395376 \\
\hline 31 & $\mathrm{~N} 2$ & N2 & B & 22 & 21 & 20 & 1.360 & 119.220 & -178.349 & -0.986087 \\
\hline 2 & HN21 & $\mathrm{H}$ & $E$ & 31 & 22 & 21 & 1.022 & 112.834 & 23.897 & 0.433155 \\
\hline 3 & HN22 & $\mathrm{H}$ & $E$ & 31 & 22 & 21 & 1.010 & 117.498 & 160.338 & 0.433155 \\
\hline 34 & H8 ' 1 & $\mathrm{H} 1$ & $\mathrm{E}$ & 18 & 17 & 16 & 1.091 & 114.107 & -45.232 & 0.081093 \\
\hline 35 & $\mathrm{H} 8$ ' 2 & $\mathrm{H} 1$ & $E$ & 18 & 17 & 16 & 1.091 & 106.981 & -166.583 & 0.081093 \\
\hline 36 & $07^{\prime}$ & 0 & $E$ & 17 & 16 & 13 & 1.222 & 122.897 & -171.324 & -0.567970 \\
\hline 37 & $C 5^{\prime}$ & $\mathrm{CT}$ & $M$ & 16 & 13 & 6 & 1.455 & 115.728 & -90.970 & -0.518870 \\
\hline 8 & H5 ' 1 & $\mathrm{H} 1$ & $E$ & 37 & 16 & 13 & 1.091 & 109.311 & 153.225 & 0.193607 \\
\hline 39 & H5 ' 2 & $\mathrm{H} 1$ & $\mathrm{E}$ & 37 & 16 & 13 & 1.093 & 109.542 & 34.125 & 0.193607 \\
\hline 10 & $C^{\prime}$ & $C$ & $M$ & 37 & 16 & 13 & 1.537 & 114.293 & -86.183 & 0.829710 \\
\hline & $01^{\prime}$ & 0 & $\mathrm{E}$ & 40 & 37 & 16 & 1.220 & 119.698 & 173.001 & -0.630603 \\
\hline
\end{tabular}

LOOP

C8 N9

C5 C4 
IMPROPER

\begin{tabular}{|c|c|c|c|}
\hline$-M$ & $C 2^{\prime}$ & $\mathrm{N} 1^{\prime}$ & $\mathrm{H} 1{ }^{\prime} 3$ \\
\hline$C 7^{\prime}$ & C3' & N4 ' & C5 ${ }^{\prime}$ \\
\hline C8' & N4 ' & C7' & $07^{\prime}$ \\
\hline C4 & $\mathrm{C} 8$ & N9 & $C 8^{\prime}$ \\
\hline C5 & N9 & C4 & N3 \\
\hline $\mathrm{N} 2$ & N1 & $\mathrm{C} 2$ & N3 \\
\hline C6 & $\mathrm{C} 2$ & N1 & HN1 \\
\hline C5 & N1 & C6 & 06 \\
\hline C6 & $\mathrm{C} 4$ & C5 & N7 \\
\hline $\mathrm{H} 8$ & N9 & $\mathrm{C} 8$ & N7 \\
\hline $\mathrm{C} 2$ & HN21 & $\mathrm{N} 2$ & HN22 \\
\hline C5 ${ }^{\prime}$ & $+M$ & $C^{\prime}$ & 01 \\
\hline
\end{tabular}


TPN Residue Topology

$0 \quad 0 \quad 2$

This is a remark line

TPN.res

TPN INT $\theta$

CORRECT OMIT DU BEG

0.0000

$\begin{array}{llllrrrrrrr}1 & \text { DUMM } & \text { DU } & \text { M } & 0 & -1 & -2 & 0.000 & .0 & .0 & .00000 \\ 2 & \text { DUMM } & \text { DU } & \text { M } & 1 & 0 & -1 & 1.449 & .0 & .0 & .00000 \\ 3 & \text { DUMM } & \text { DU } & \text { M } & 2 & 1 & 0 & 1.523 & 111.21 & .0 & .00000\end{array}$

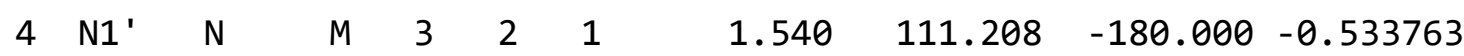

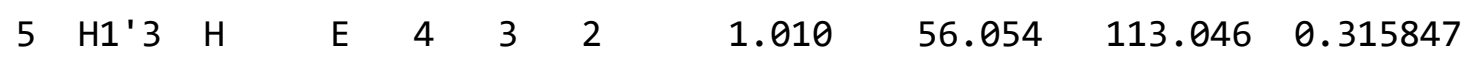

$\begin{array}{lllllllllll}6 & \text { C2 }^{\prime} & \text { CT } & \text { M } & 4 & 3 & 2 & 1.455 & 137.697 & 17.473 & -0.029240\end{array}$

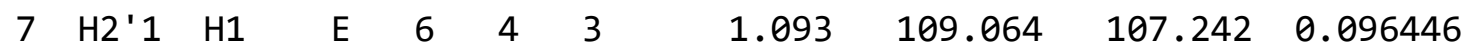

$\begin{array}{llllllllll}8 & H & H & \prime\end{array}$

$\begin{array}{lllllllllll}9 & \text { C3 }^{\prime} & \text { CT } & \text { M } & 6 & 4 & 3 & 1.538 & 114.143 & -13.070 & 0.020016\end{array}$

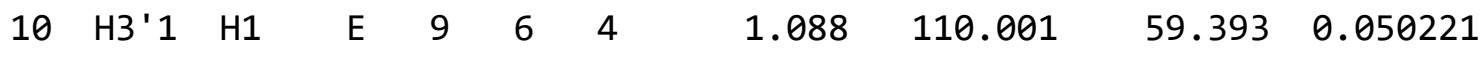

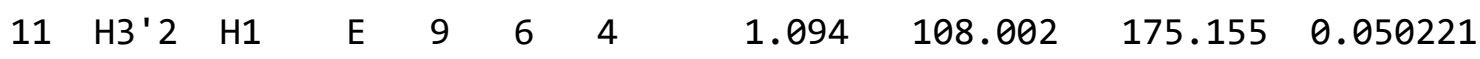

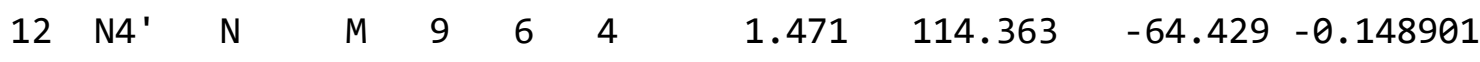

$\begin{array}{lllllllllll}13 & \text { C7 }^{\prime} & \text { C } & \text { B } & 12 & 9 & 6 & 1.366 & 125.003 & 101.819 & 0.628924\end{array}$

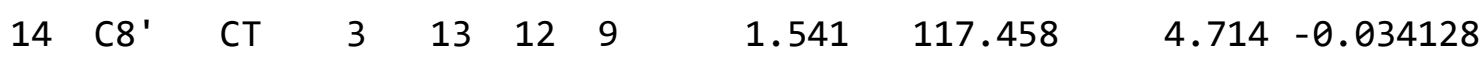

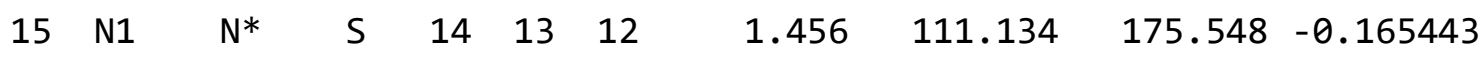

$\begin{array}{lllllllllll}16 & \text { C2 } & \text { C } & \text { B } & 15 & 14 & 13 & 1.386 & 117.868 & -85.270 & 0.735067\end{array}$

$\begin{array}{lllllllllll}17 & 02 & 0 & E & 16 & 15 & 14 & 1.223 & 123.280 & -3.157 & -0.597092\end{array}$

$\begin{array}{lllllllllll}18 & N 3 & \text { NA } & \text { B } & 16 & 15 & 14 & 1.380 & 114.019 & 177.470 & -0.670135\end{array}$ 


\begin{tabular}{|c|c|c|c|c|c|c|c|c|c|c|}
\hline 19 & $\mathrm{C} 4$ & C & B & 18 & 16 & 15 & 1.398 & 128.006 & -2.192 & 0.738982 \\
\hline 20 & 04 & 0 & $E$ & 19 & 18 & 16 & 1.228 & 119.922 & -179.240 & -0.588986 \\
\hline 21 & $\mathrm{C} 5$ & CM & B & 19 & 18 & 16 & 1.455 & 114.522 & 0.798 & -0.028144 \\
\hline 22 & C5M & $\mathrm{CT}$ & 3 & 21 & 19 & 18 & 1.502 & 118.934 & -179.975 & -0.440748 \\
\hline 23 & HM51 & $\mathrm{HC}$ & $E$ & 22 & 21 & 19 & 1.093 & 110.992 & -59.552 & 0.140170 \\
\hline 24 & HM52 & $\mathrm{HC}$ & E & 22 & 21 & 19 & 1.090 & 110.813 & 179.902 & 0.140170 \\
\hline 25 & HM53 & $\mathrm{HC}$ & $\mathrm{E}$ & 22 & 21 & 19 & 1.094 & 110.948 & 59.430 & 0.140170 \\
\hline 26 & $\mathrm{C} 6$ & CM & $S$ & 21 & 19 & 18 & 1.353 & 117.919 & 0.075 & -0.182205 \\
\hline 27 & $\mathrm{H} 6$ & $\mathrm{H} 4$ & $\mathrm{E}$ & 26 & 21 & 19 & 1.082 & 121.362 & -179.850 & 0.227546 \\
\hline 28 & HN3 & $\mathrm{H}$ & $E$ & 18 & 16 & 15 & 1.013 & 115.370 & 179.371 & 0.390831 \\
\hline 29 & H8 ' 1 & $\mathrm{H} 1$ & $\mathrm{E}$ & 14 & 13 & 12 & 1.088 & 110.562 & -63.921 & 0.057266 \\
\hline 30 & $\mathrm{H} 8^{\prime} 2$ & $\mathrm{H} 1$ & E & 14 & 13 & 12 & 1.089 & 110.317 & 55.742 & 0.057266 \\
\hline 31 & $07^{\prime}$ & 0 & $\mathrm{E}$ & 13 & 12 & 9 & 1.226 & 122.290 & -176.448 & -0.550788 \\
\hline 32 & $\mathrm{C} 5^{\prime}$ & $\mathrm{CT}$ & M & 12 & 9 & 6 & 1.463 & 117.070 & -72.372 & -0.571611 \\
\hline 33 & H5 ' 1 & $\mathrm{H} 1$ & $\mathrm{E}$ & 32 & 12 & 9 & 1.090 & 109.279 & 145.003 & 0.229626 \\
\hline 34 & $\mathrm{H} 5$ ' 2 & $\mathrm{H} 1$ & $\mathrm{E}$ & 32 & 12 & 9 & 1.090 & 109.263 & 27.830 & 0.229626 \\
\hline 35 & $C^{\prime}$ & C & M & 32 & 12 & 9 & 1.533 & 116.828 & -93.586 & 0.827910 \\
\hline 36 & $01^{\prime}$ & 0 & $\mathrm{E}$ & 35 & 32 & 12 & 1.233 & 118.842 & 177.631 & -0.631565 \\
\hline
\end{tabular}

LOOP

C6 N1

IMPROPER

$\begin{array}{cccc}-\mathrm{M} & \mathrm{C} 2^{\prime} & \mathrm{N} 1^{\prime} & \mathrm{H} 1^{\prime} 3 \\ \mathrm{C}^{\prime} & \mathrm{C} 5^{\prime} & \mathrm{N} 4^{\prime} & \mathrm{C} 3^{\prime} \\ \mathrm{C} 8^{\prime} & \mathrm{N} 4{ }^{\prime} & \mathrm{C} 7^{\prime} & \mathrm{O} 7^{\prime} \\ \mathrm{C} 2 & \mathrm{C} 6 & \mathrm{~N} 1 & \mathrm{C} 8^{\prime}\end{array}$ 


$\begin{array}{rrrr}\text { N1 } & \text { N3 } & \text { C2 } & \text { O2 } \\ \text { C2 } & \mathrm{C} 4 & \text { N3 } & \text { HN3 } \\ \text { C5 } & \text { N3 } & \mathrm{C} 4 & 04 \\ \text { C4 } & \mathrm{C} 6 & \mathrm{C} 5 & \mathrm{C} 5 \mathrm{M} \\ \mathrm{C} 5 & \mathrm{H} 6 & \mathrm{C} 6 & \mathrm{~N} 1 \\ \mathrm{C}^{\prime} & +\mathrm{M} & \mathrm{C}^{\prime} & 01^{\prime}\end{array}$

DONE

STOP 
TPR Residue Topology

$0 \quad 0 \quad 2$

This is a remark line

TPR.res

TPR INT 0

CORRECT OMIT DU BEG

0.0000

$\begin{array}{llllrrrrrrr}1 & \text { DUMM } & \text { DU } & \text { M } & 0 & -1 & -2 & 0.000 & .0 & .0 & .00000 \\ 2 & \text { DUMM } & \text { DU } & \text { M } & 1 & 0 & -1 & 1.449 & .0 & .0 & .00000 \\ 3 & \text { DUMM } & \text { DU } & \text { M } & 2 & 1 & 0 & 1.523 & 111.21 & .0 & .00000\end{array}$

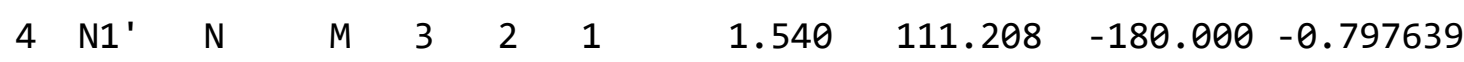

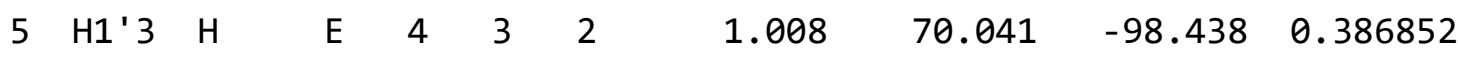

$\begin{array}{lllllllllll}6 & \mathrm{C}^{\prime} & \text { CT } & \text { M } & 4 & 3 & 2 & 1.457 & 78.039 & 136.848 & 0.339673\end{array}$

$\begin{array}{lllllllllll}7 & \mathrm{C} 01 & \mathrm{CT} & 3 & 6 & 4 & 3 & 1.535 & 111.524 & -64.834 & 0.052932\end{array}$

$\begin{array}{lllllllllll}8 & 001 & \mathrm{OH} & \mathrm{S} & 7 & 6 & 4 & 1.411 & 111.670 & 179.654 & -0.672520\end{array}$

$\begin{array}{lllllllllll}9 & \mathrm{H} 01 & \mathrm{HO} & \mathrm{E} & 8 & 7 & 6 & 0.965 & 108.390 & -70.261 & 0.429370\end{array}$

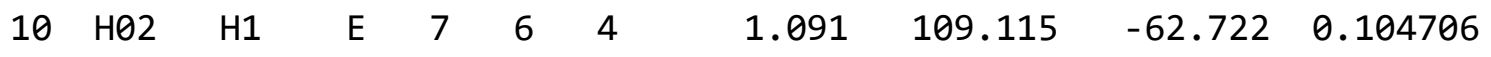

$\begin{array}{lllllllllll}11 & \mathrm{H} 04 & \mathrm{H} 1 & \mathrm{E} & 7 & 6 & 4 & 1.099 & 109.132 & 55.095 & 0.104706\end{array}$

$\begin{array}{llllllllll}12 & \mathrm{H} 2 & \end{array}$

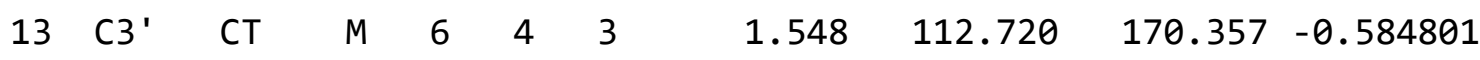

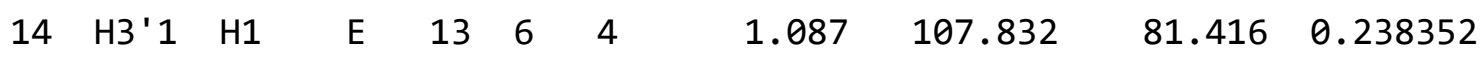

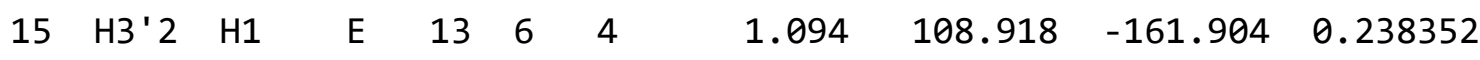

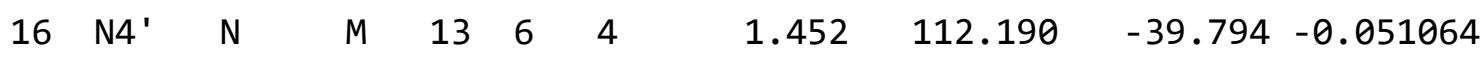

$\begin{array}{lllllllllll}17 & \text { C7 }^{\prime} & \text { C } & \text { B } & 16 & 13 & 6 & 1.366 & 125.088 & 114.801 & 0.580577\end{array}$

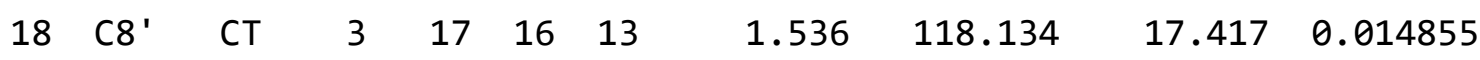




\begin{tabular}{|c|c|c|c|c|c|c|c|c|c|c|}
\hline 19 & $\mathrm{~N} 1$ & $\mathrm{~N}^{*}$ & $\mathrm{~S}$ & 18 & 17 & 16 & 1.450 & 108.353 & 143.999 & -0.167300 \\
\hline 20 & $\mathrm{C} 2$ & C & B & 19 & 18 & 17 & 1.380 & 115.745 & -66.686 & 0.743678 \\
\hline 21 & 02 & 0 & $E$ & 20 & 19 & 18 & 1.224 & 122.692 & -4.416 & -0.573710 \\
\hline 22 & N3 & NA & B & 20 & 19 & 18 & 1.372 & 114.365 & 176.475 & -0.691734 \\
\hline 23 & $\mathrm{C} 4$ & $C$ & B & 22 & 20 & 19 & 1.403 & 127.848 & -4.813 & 0.749811 \\
\hline 24 & 04 & 0 & $E$ & 23 & 22 & 20 & 1.216 & 120.374 & -178.615 & -0.582582 \\
\hline 25 & $\mathrm{C} 5$ & CM & $\mathrm{B}$ & 23 & 22 & 20 & 1.461 & 113.950 & 1.191 & -0.026380 \\
\hline 26 & C5M & CT & 3 & 25 & 23 & 22 & 1.498 & 117.733 & -178.862 & -0.437495 \\
\hline 27 & HM51 & $\mathrm{HC}$ & $E$ & 26 & 25 & 23 & 1.095 & 110.672 & -59.242 & 0.138458 \\
\hline 28 & HM52 & $\mathrm{HC}$ & $E$ & 26 & 25 & 23 & 1.093 & 111.190 & 179.815 & 0.138458 \\
\hline 29 & HM53 & $\mathrm{HC}$ & $E$ & 26 & 25 & 23 & 1.095 & 110.584 & 59.067 & 0.138458 \\
\hline 30 & C6 & $\mathrm{CM}$ & $\mathrm{S}$ & 25 & 23 & 22 & 1.346 & 118.318 & 0.647 & -0.186947 \\
\hline 31 & $\mathrm{H} 6$ & $\mathrm{H} 4$ & $E$ & 30 & 25 & 23 & 1.085 & 121.840 & -179.350 & 0.227723 \\
\hline 32 & HN3 & $\mathrm{H}$ & $E$ & 22 & 20 & 19 & 1.012 & 115.688 & 179.349 & 0.392879 \\
\hline 33 & H8 ' 1 & $\mathrm{H} 1$ & $E$ & 18 & 17 & 16 & 1.092 & 109.913 & -95.842 & 0.089868 \\
\hline 34 & H8 ' 2 & $\mathrm{H} 1$ & $E$ & 18 & 17 & 16 & 1.090 & 112.164 & 24.961 & 0.089868 \\
\hline 35 & $07^{\prime}$ & 0 & $E$ & 17 & 16 & 13 & 1.222 & 122.701 & -166.868 & -0.572954 \\
\hline 36 & $C 5^{\prime}$ & $\mathrm{CT}$ & $M$ & 16 & 13 & 6 & 1.450 & 116.836 & -64.723 & -0.368505 \\
\hline 37 & H5 ' 1 & $\mathrm{H} 1$ & $E$ & 36 & 16 & 13 & 1.092 & 107.728 & 168.175 & 0.147145 \\
\hline 38 & H5 ' 2 & $\mathrm{H} 1$ & $E$ & 36 & 16 & 13 & 1.093 & 111.834 & 49.589 & 0.147145 \\
\hline 39 & $C^{\prime}$ & $C$ & $M$ & 36 & 16 & 13 & 1.533 & 113.024 & -71.280 & 0.837001 \\
\hline 10 & $01^{\prime}$ & 0 & $E$ & 39 & 36 & 16 & 1.223 & 120.454 & 147.829 & -0.655196 \\
\hline
\end{tabular}

LOOP

C6 N1

\section{IMPROPER}




$\begin{array}{cccc}-\mathrm{M} & \mathrm{C}^{\prime} & \mathrm{N} 1^{\prime} & \mathrm{H} 1^{\prime} 3 \\ \mathrm{C} 7^{\prime} & \mathrm{C}^{\prime} & \mathrm{N}^{\prime} & \mathrm{C} 5^{\prime} \\ \mathrm{C} 8^{\prime} & \mathrm{N} 4^{\prime} & \mathrm{C} 7^{\prime} & \mathrm{O} 7^{\prime} \\ \mathrm{C} 2 & \mathrm{C} 6 & \mathrm{~N} 1 & \mathrm{C} 8^{\prime} \\ \mathrm{N} 1 & \mathrm{~N} 3 & \mathrm{C} 2 & \mathrm{O} 2 \\ \mathrm{C} 2 & \mathrm{C} 4 & \mathrm{~N} 3 & \mathrm{HN} 3 \\ \mathrm{C} 5 & \mathrm{~N} 3 & \mathrm{C} 4 & \mathrm{O} 4 \\ \mathrm{C} 4 & \mathrm{C} 6 & \mathrm{C} 5 & \mathrm{C} 5 \mathrm{M} \\ \mathrm{C} 5 & \mathrm{H} 6 & \mathrm{C} 6 & \mathrm{~N} 1 \\ \mathrm{C} 5 & +\mathrm{M} & \mathrm{C}^{\prime} & 01^{\prime}\end{array}$

DONE

STOP 
QPC Residue Topology

$\begin{array}{lll}0 & 0 & 2\end{array}$

This is a remark line

QPC.res

QPC INT 0

CORRECT OMIT DU BEG

0.0000

$\begin{array}{llllrrrrrrr}1 & \text { DUMM } & \text { DU } & \text { M } & 0 & -1 & -2 & 0.000 & .0 & .0 & .00000 \\ 2 & \text { DUMM } & \text { DU } & \text { M } & 1 & 0 & -1 & 1.449 & .0 & .0 & .00000 \\ 3 & \text { DUMM } & \text { DU } & \text { M } & 2 & 1 & 0 & 1.523 & 111.21 & .0 & .00000\end{array}$

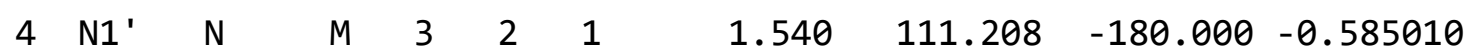

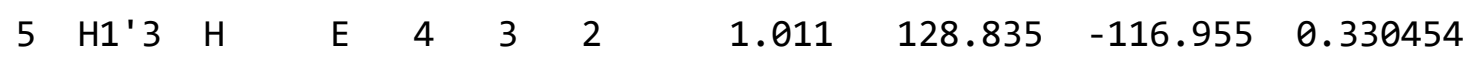

$\begin{array}{lllllllllll}6 & \text { C2 }^{\prime} & \text { CT } & \text { M } & 4 & 3 & 2 & 1.454 & 47.446 & -22.109 & -0.026374\end{array}$

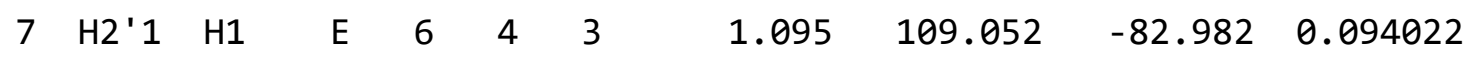

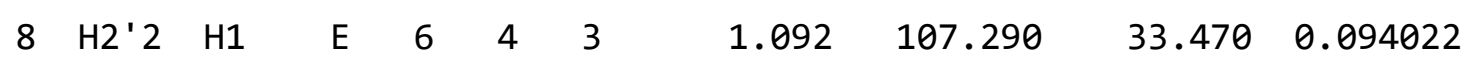

$\begin{array}{lllllllllll}9 & \text { C3 }^{\prime} & \text { CT } & \text { M } & 6 & 4 & 3 & 1.540 & 114.191 & 156.461 & 0.105621\end{array}$

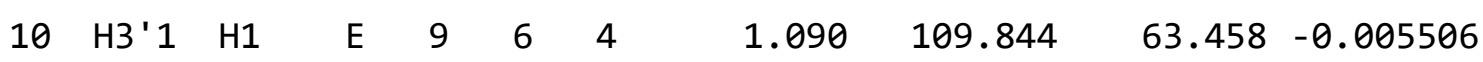

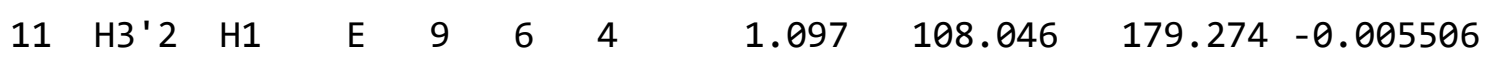

$\begin{array}{lllllllllll}12 & N^{\prime} & N & M & 9 & 6 & 4 & 1.467 & 114.608 & -60.105 & -0.078921\end{array}$

$\begin{array}{lllllllllll}13 & \mathrm{C}^{\prime} & \text { C } & \text { B } & 12 & 9 & 6 & 1.377 & 124.802 & 100.745 & 0.577156\end{array}$

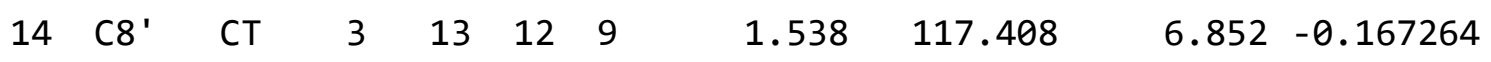

$\begin{array}{lllllllllll}15 & \text { C7 } & \text { CA } & \text { S } & 14 & 13 & 12 & 1.509 & 112.155 & 178.410 & 0.096671\end{array}$

$\begin{array}{lllllllllll}16 & \text { C4 } & \text { CA } & \text { S } & 15 & 14 & 13 & 1.430 & 121.497 & -79.924 & 0.122050\end{array}$

$\begin{array}{lllllllllll}17 & \text { C3 } & \text { CA } & \text { B } & 16 & 15 & 14 & 1.419 & 125.420 & 0.720 & -0.404238\end{array}$

$\begin{array}{lllllllllll}18 & \text { C2 } & \text { CA } & \text { B } & 17 & 16 & 15 & 1.385 & 119.430 & -179.390 & -0.058922\end{array}$ 


\begin{tabular}{|c|c|c|c|c|c|c|c|c|c|c|}
\hline 19 & $\mathrm{C} 1$ & $\mathrm{CA}$ & B & 18 & 17 & 16 & 1.412 & 122.604 & -0.263 & -0.375315 \\
\hline 20 & $\mathrm{C} 9$ & $\mathrm{CA}$ & B & 19 & 18 & 17 & 1.394 & 120.339 & 0.016 & 0.434954 \\
\hline 21 & 01 & OS & $E$ & 20 & 19 & 18 & 1.320 & 124.723 & -179.355 & -0.749390 \\
\hline 22 & $\mathrm{C} 5$ & $C A$ & $S$ & 20 & 19 & 18 & 1.438 & 117.105 & 0.057 & 0.075139 \\
\hline 23 & N1 & $\mathrm{N}^{*}$ & $S$ & 22 & 20 & 19 & 1.366 & 114.484 & 179.622 & -0.291834 \\
\hline 24 & $\mathrm{C} 8$ & $C A$ & B & 23 & 22 & 20 & 1.325 & 119.480 & -179.875 & 0.045994 \\
\hline 25 & $\mathrm{C} 6$ & $C A$ & $S$ & 24 & 23 & 22 & 1.406 & 121.618 & 0.739 & -0.268322 \\
\hline 26 & $\mathrm{H} 6$ & $\mathrm{HA}$ & $E$ & 25 & 24 & 23 & 1.086 & 118.814 & 179.257 & 0.168902 \\
\hline 27 & $\mathrm{H} 5$ & $\mathrm{H} 4$ & $E$ & 24 & 23 & 22 & 1.085 & 116.513 & -179.432 & 0.163078 \\
\hline 28 & $\mathrm{H} 1$ & $\mathrm{HA}$ & $E$ & 19 & 18 & 17 & 1.086 & 120.399 & -179.883 & 0.190845 \\
\hline 29 & $\mathrm{H} 2$ & $\mathrm{HA}$ & $E$ & 18 & 17 & 16 & 1.087 & 118.991 & 179.908 & 0.154272 \\
\hline 30 & $\mathrm{H} 3$ & $\mathrm{HA}$ & $E$ & 17 & 16 & 15 & 1.084 & 120.583 & 0.730 & 0.196832 \\
\hline 31 & H01 & $\mathrm{HC}$ & E & 14 & 13 & 12 & 1.096 & 109.658 & 55.866 & 0.070805 \\
\hline 32 & $\mathrm{HO2}$ & $\mathrm{HC}$ & $E$ & 14 & 13 & 12 & 1.093 & 108.962 & -60.351 & 0.070805 \\
\hline 33 & $07^{\prime}$ & 0 & $E$ & 13 & 12 & 9 & 1.230 & 121.811 & -174.122 & -0.554091 \\
\hline 34 & $\mathrm{C5}{ }^{\prime}$ & CT & M & 12 & 9 & 6 & 1.461 & 116.646 & -70.490 & -0.480610 \\
\hline 35 & H5 ' 1 & $\mathrm{H} 1$ & $E$ & 34 & 12 & 9 & 1.092 & 107.430 & 163.054 & 0.199618 \\
\hline 36 & $\mathrm{H} 5$ ' 2 & $\mathrm{H} 1$ & $E$ & 34 & 12 & 9 & 1.093 & 110.344 & 46.385 & 0.199618 \\
\hline 37 & $C^{\prime}$ & C & M & 34 & 12 & 9 & 1.533 & 116.136 & -76.540 & 0.785215 \\
\hline 38 & $01^{\prime}$ & 0 & $E$ & 37 & 34 & 12 & 1.233 & 119.447 & 163.388 & -0.624769 \\
\hline
\end{tabular}

LOOP

C6 C7

C5 C4

IMPROPER

C2' H1' $^{\prime} 3 \quad \mathrm{N1} 1^{\prime} \quad-M$ 


$\begin{array}{llll}\mathrm{C}^{\prime} & \mathrm{C}^{\prime} & \mathrm{N}^{\prime} & \mathrm{C}^{\prime} \\ \mathrm{C}{ }^{\prime} & \mathrm{N} 4^{\prime} & \mathrm{C}^{\prime} & \mathrm{O}^{\prime} \\ \mathrm{C} 4 & \mathrm{C} 6 & \mathrm{C} 7 & \mathrm{C}^{\prime} \\ \mathrm{C} 7 & \mathrm{C} 3 & \mathrm{C} 4 & \mathrm{C} 5 \\ \mathrm{C} 4 & \mathrm{C} 2 & \mathrm{C} 3 & \mathrm{H} 3 \\ \mathrm{C} 3 & \mathrm{C} 1 & \mathrm{C} 2 & \mathrm{H} 2 \\ \mathrm{C} 2 & \mathrm{C} 9 & \mathrm{C} 1 & \mathrm{H} 1 \\ \mathrm{C} 1 & \mathrm{C}^{\prime} & \mathrm{C} 9 & \mathrm{O} 1 \\ \mathrm{C} 4 & \mathrm{C} 9 & \mathrm{C} 5 & \mathrm{~N} 1 \\ \mathrm{C} 6 & \mathrm{H} 5 & \mathrm{C} 8 & \mathrm{~N} 1 \\ \mathrm{C} 7 & \mathrm{C} 8 & \mathrm{C} 6 & \mathrm{H} 6 \\ \mathrm{C} 5 & \mathrm{O} 1^{\prime} & \mathrm{C}^{\prime} & +\mathrm{M}\end{array}$

DONE

STOP 
QPN Residue Topology

$0 \quad 0 \quad 2$

This is a remark line

QPN.res

QPN INT 0

CORRECT OMIT DU BEG

0.0000

$\begin{array}{llllrrrrrrr}1 & \text { DUMM } & \text { DU } & \text { M } & 0 & -1 & -2 & 0.000 & .0 & .0 & .00000 \\ 2 & \text { DUMM } & \text { DU } & \text { M } & 1 & 0 & -1 & 1.449 & .0 & .0 & .00000 \\ 3 & \text { DUMM } & \text { DU } & \text { M } & 2 & 1 & 0 & 1.523 & 111.21 & .0 & .00000\end{array}$

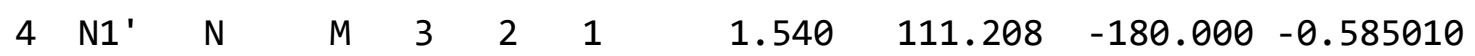

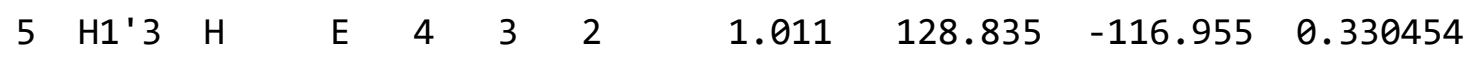

$\begin{array}{lllllllllll}6 & \text { C2 }^{\prime} & \text { CT } & \text { M } & 4 & 3 & 2 & 1.454 & 47.446 & -22.109 & -0.026374\end{array}$

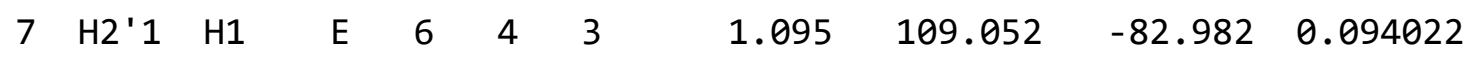

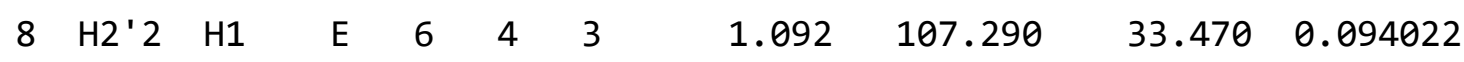

$\begin{array}{lllllllllll}9 & \text { C3 }^{\prime} & \text { CT } & \text { M } & 6 & 4 & 3 & 1.540 & 114.191 & 156.461 & 0.105621\end{array}$

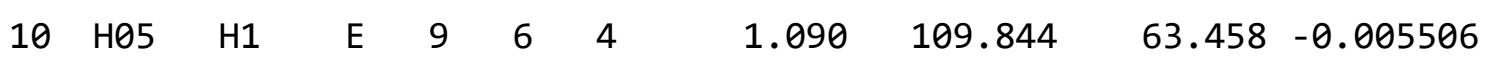

$\begin{array}{lllllllllll}11 & H 06 & H & \text { E } & 9 & 6 & 4 & 1.097 & 108.046 & 179.274 & -0.005506\end{array}$

$\begin{array}{lllllllllll}12 & N^{\prime} & N & M & 9 & 6 & 4 & 1.467 & 114.608 & -60.105 & -0.078921\end{array}$

$\begin{array}{lllllllllll}13 & \mathrm{C}^{\prime} & \text { C } & \text { B } & 12 & 9 & 6 & 1.377 & 124.802 & 100.745 & 0.577156\end{array}$

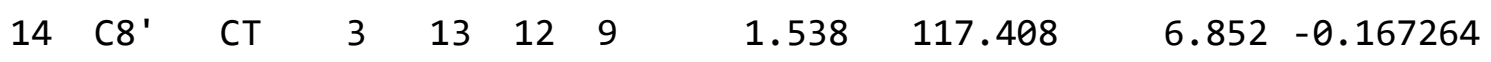

$\begin{array}{lllllllllll}15 & \text { C7 } & \text { CA } & \text { S } & 14 & 13 & 12 & 1.509 & 112.155 & 178.410 & 0.096671\end{array}$

$\begin{array}{lllllllllll}16 & \text { C4 } & \text { CA } & \text { S } & 15 & 14 & 13 & 1.430 & 121.497 & -79.924 & 0.122050\end{array}$

$\begin{array}{lllllllllll}17 & \text { C3 } & \text { CA } & \text { B } & 16 & 15 & 14 & 1.419 & 125.420 & 0.720 & -0.404238\end{array}$

$\begin{array}{lllllllllll}18 & \text { C2 } & \text { CA } & \text { B } & 17 & 16 & 15 & 1.385 & 119.430 & -179.390 & -0.058922\end{array}$ 


\begin{tabular}{|c|c|c|c|c|c|c|c|c|c|c|}
\hline 19 & $\mathrm{C} 1$ & $\mathrm{CA}$ & B & 18 & 17 & 16 & 1.412 & 122.604 & -0.263 & -0.375315 \\
\hline 20 & $\mathrm{C9}$ & $C A$ & B & 19 & 18 & 17 & 1.394 & 120.339 & 0.016 & 0.434954 \\
\hline 21 & 01 & OT & $E$ & 20 & 19 & 18 & 1.320 & 124.723 & -179.355 & -0.749390 \\
\hline 22 & $\mathrm{C} 5$ & $C A$ & $S$ & 20 & 19 & 18 & 1.438 & 117.105 & 0.057 & 0.075139 \\
\hline 23 & N1 & $N X$ & $S$ & 22 & 20 & 19 & 1.366 & 114.484 & 179.622 & -0.291834 \\
\hline 24 & $\mathrm{C} 8$ & CA & B & 23 & 22 & 20 & 1.325 & 119.480 & -179.875 & 0.045994 \\
\hline 25 & $\mathrm{C} 6$ & CA & $S$ & 24 & 23 & 22 & 1.406 & 121.618 & 0.739 & -0.268322 \\
\hline 26 & H01 & $\mathrm{HA}$ & $E$ & 25 & 24 & 23 & 1.086 & 118.814 & 179.257 & 0.168902 \\
\hline 27 & $\mathrm{H} 07$ & $\mathrm{H} 4$ & $E$ & 24 & 23 & 22 & 1.085 & 116.513 & -179.432 & 0.163078 \\
\hline 28 & $\mathrm{H} 1$ & $\mathrm{HA}$ & $E$ & 19 & 18 & 17 & 1.086 & 120.399 & -179.883 & 0.190845 \\
\hline 29 & $\mathrm{H} 2$ & $\mathrm{HA}$ & $E$ & 18 & 17 & 16 & 1.087 & 118.991 & 179.908 & 0.1542 \\
\hline 30 & $\mathrm{H} 08$ & $\mathrm{HA}$ & $E$ & 17 & 16 & 15 & 1.084 & 120.583 & 0.730 & 0.196832 \\
\hline 31 & HO2 & $\mathrm{HC}$ & E & 14 & 13 & 12 & 1.096 & 109.658 & 55.866 & 0.070805 \\
\hline 32 & H09 & $\mathrm{HC}$ & $E$ & 14 & 13 & 12 & 1.093 & 108.962 & -60.351 & 0.070805 \\
\hline 33 & $07^{\prime}$ & 0 & $E$ & 13 & 12 & 9 & 1.230 & 121.811 & -174.122 & -0.554091 \\
\hline 34 & C5 ${ }^{\prime}$ & CT & M & 12 & 9 & 6 & 1.461 & 116.646 & -70.490 & -0.480610 \\
\hline 35 & $\mathrm{H} 03$ & $\mathrm{H} 1$ & $E$ & 34 & 12 & 9 & 1.092 & 107.430 & 163.054 & 0.199618 \\
\hline 36 & $\mathrm{HO4}$ & $\mathrm{H} 1$ & $E$ & 34 & 12 & 9 & 1.093 & 110.344 & 46.385 & 0.199618 \\
\hline 37 & $C^{\prime}$ & $C$ & M & 34 & 12 & 9 & 1.533 & 116.136 & -76.540 & 0.785215 \\
\hline 38 & $01^{\prime}$ & 0 & $E$ & 37 & 34 & 12 & 1.233 & 119.447 & 163.388 & -0.624769 \\
\hline
\end{tabular}

LOOP

C6 C7

C5 C4

IMPROPER

C2' H1' $^{\prime} 3 \quad \mathrm{N1} 1^{\prime} \quad-M$ 


\begin{tabular}{|c|c|c|c|}
\hline$C 7^{\prime}$ & $\mathrm{C} 5^{\prime}$ & N4 ' & $\mathrm{C} 3$ \\
\hline C8' & N4 ' & C7' & 07 \\
\hline C4 & C6 & C7 & C8 \\
\hline C7 & C3 & C4 & $C$ \\
\hline C4 & C2 & C3 & HO\& \\
\hline C3 & C1 & $C 2$ & $\mathrm{H}_{2}^{2}$ \\
\hline $\mathrm{C} 2$ & C9 & C1 & t]. \\
\hline C1 & C5 & C9 & 01 \\
\hline C4 & C9 & C5 & \\
\hline C6 & H07 & C8 & \\
\hline C7 & C8 & C6 & \\
\hline$C 5^{\prime}$ & $01^{\prime}$ & $C^{\prime}$ & \\
\hline
\end{tabular}

DONE

STOP 
Cu Residue Topology

$0 \quad 0 \quad 2$

This is a remark line

Cu.res

$\mathrm{CU}$ INT $\theta$

CORRECT OMIT DU BEG

0.0000

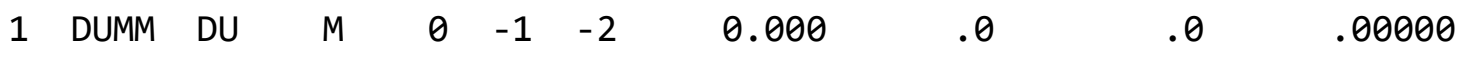

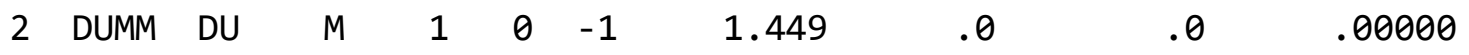

$\begin{array}{llllllllll}3 & \text { DUMM DU } & \text { M } & 2 & 1 & 0 & 1.523 & 111.21 & .0 & .00000\end{array}$

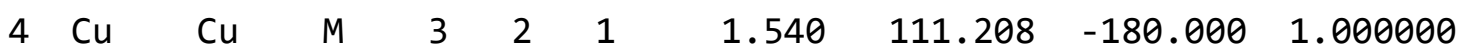

LOOP

IMPROPER

DONE

STOP 
Additional Force Field Parameters for APN

Remark line goes here

MASS

BOND

ANGLE

C - $\mathrm{CT}-\mathrm{N}^{*} \quad 68.100 \quad 106.510$ same as ca-c3-nc, penalty score $=2.5$

DIHE

IMPROPER

C $-\mathrm{CT}-\mathrm{N}-\mathrm{CT}$

1.0

180.0

2.0

$\mathrm{CT}-\mathrm{N}-\mathrm{C}-\mathrm{O}$

10.5

180.0

2.0

$\mathrm{CB}-\mathrm{N} *-\mathrm{CB}-\mathrm{NC}$

1.1

180.0

2.0

H5-NC-CQ-NC

1.1

180.0

2.0

CA-CB-CB-NB

1.1

180.0

2.0

H5-N*-CK-NB

1.1

180.0

2.0

$\mathrm{CA}-\mathrm{H}-\mathrm{N} 2-\mathrm{H}$

1.0

180.0

2.0

NONBON 
Additional Force Field Parameters for APR

Remark line goes here

MASS

BOND

ANGLE

C - $\mathrm{CT}-\mathrm{N}^{*} \quad 68.100 \quad 106.510$ same as ca-c3-nc, penalty score $=2.5$

DIHE

IMPROPER

C $-\mathrm{CT}-\mathrm{N}-\mathrm{CT}$

1.1

180.0

2.0

Using the default

value

CT-N $-\mathrm{C}-\mathrm{O}$

1.1

180.0

2.0

Using the default

value

$\mathrm{CB}-\mathrm{N}^{*}-\mathrm{CB}-\mathrm{NC}$

1.1

180.0

2.0

Using the default

value

H5-NC-CQ-NC

1.1

180.0

2.0

Using the default

value

CA-CB-CB-NB

1.1

180.0

2.0

Using the default

value

1.1

180.0

2.0

Using the default

value

$\mathrm{CA}-\mathrm{H}-\mathrm{N} 2-\mathrm{H}$

value

1.1

180.0

2.0

Using the default

NONBON 
Additional Force Field Parameters for CPN

Remark line goes here

MASS

BOND

ANGLE

C - $\mathrm{CT}-\mathrm{N}^{*} \quad 68.100 \quad 106.510$ same as ca-c3-nc, penalty score $=2.5$

DIHE

IMPROPER

C $-\mathrm{CT}-\mathrm{N}-\mathrm{CT}$

1.0

180.0

2.0

$\mathrm{CT}-\mathrm{N}-\mathrm{C}-\mathrm{O}$

10.5

180.0

2.0

$\mathrm{N}^{*}-\mathrm{NC}-\mathrm{C}-\mathrm{O}$

10.5

180.0

2.0

$\mathrm{CA}-\mathrm{H}-\mathrm{N} 2-\mathrm{H}$

1.0

180.0

2.0

CA-CM-CM-HA

1.1

180.0

2.0

CM-H4-CM-N*

1.1

180.0

2.0

NONBON 
Additional Force Field Parameters for CPR

Remark line goes here

MASS

BOND

ANGLE

C -CT-N* $68.100 \quad 106.510$ same as ca-c3-nc, penalty score $=2.5$

DIHE

IMPROPER

$\begin{array}{lcccc}\begin{array}{l}\text { C }-\mathrm{CT}-\mathrm{N}-\mathrm{CT} \\ \text { value }\end{array} & 1.1 & 180.0 & 2.0 & \text { Using the default } \\ \begin{array}{l}\text { CT-N -C -O } \\ \text { value }\end{array} & 1.1 & 180.0 & 2.0 & \text { Using the default } \\ \begin{array}{l}\mathrm{N}^{*}-\mathrm{NC}-\mathrm{C}-\mathrm{O} \\ \text { value }\end{array} & 1.1 & 180.0 & 2.0 & \text { Using the default } \\ \begin{array}{l}\text { CA-H -N2-H } \\ \text { value }\end{array} & 1.1 & 180.0 & 2.0 & \text { Using the default } \\ \begin{array}{l}\text { CA-CM-CM-HA } \\ \text { value }\end{array} & 1.1 & 180.0 & 2.0 & \text { Using the default } \\ \begin{array}{l}\text { CM-H4-CM-N* } \\ \text { value }\end{array} & 1.1 & 180.0 & 2.0 & \text { Using the default }\end{array}$

value

NONBON 
Additional Force Field Parameters for GPN

Remark line goes here

MASS

BOND

ANGLE

C - $\mathrm{CT}-\mathrm{N}^{*} \quad 68.100 \quad 106.510$ same as ca-c3-nc, penalty score $=2.5$

DIHE

IMPROPER

C $-\mathrm{CT}-\mathrm{N}-\mathrm{CT}$

1.0

180.0

2.0

$\mathrm{CT}-\mathrm{N}-\mathrm{C}-\mathrm{O}$

10.5

180.0

2.0

$\mathrm{CB}-\mathrm{N}^{*}-\mathrm{CB}-\mathrm{NC}$

1.1

180.0

2.0

C -CA-NA-H

1.0

180.0

2.0

CB-NA-C -O

10.5

180.0

2.0

C $-\mathrm{CB}-\mathrm{CB}-\mathrm{NB}$

1.1

180.0

2.0

$\mathrm{H}-\mathrm{N}^{*}-\mathrm{CK}-\mathrm{NB}$

1.1

180.0

2.0

$\mathrm{CA}-\mathrm{H}-\mathrm{N} 2-\mathrm{H}$

1.0

180.0

2.0

NONBON 
Additional Force Field Parameters for GPR

Remark line goes here

MASS

BOND

ANGLE

C - $-\mathrm{CT}-\mathrm{N}^{*} \quad 68.100 \quad 106.510$ same as ca-c3-nc, penalty score $=2.5$

DIHE

IMPROPER

C $-\mathrm{CT}-\mathrm{N}-\mathrm{CT}$

1.1

180.0

2.0

Using the default

value

$\mathrm{CT}-\mathrm{N}-\mathrm{C}-\mathrm{O}$

1.1

180.0

2.0

Using the default

value

$\mathrm{CB}-\mathrm{N} *-\mathrm{CB}-\mathrm{NC}$

1.1

180.0

2.0

Using the default

value

C -CA-NA-H

1.1

180.0

2.0

Using the default

value

CB-NA-C - 0

1.1

180.0

2.0

Using the default

value

1.1

180.0

2.0

Using the default

value

$\mathrm{H} 5-\mathrm{N} *-\mathrm{CK}-\mathrm{NB}$

1.1

180.0

2.0

Using the default

value

$\mathrm{CA}-\mathrm{H}-\mathrm{N} 2-\mathrm{H}$

1.1

180.0

2.0

Using the default

NONBON 
Additional Force Field Parameters for TPN

Remark line goes here

MASS

BOND

ANGLE

C - $\mathrm{CT}-\mathrm{N}^{*} \quad 68.100 \quad 106.510$ same as ca-c3-nc, penalty score $=2.5$

DIHE

IMPROPER

C $-\mathrm{CT}-\mathrm{N}-\mathrm{CT}$

1.0

180.0

2.0

$\mathrm{CT}-\mathrm{N}-\mathrm{C}-\mathrm{O}$

10.5

180.0

2.0

$N^{*}-N A-C-O$

10.5

180.0

2.0

C -C -NA-H

1.0

180.0

2.0

CM-NA-C $-O$

10.5

180.0

2.0

CM-H4-CM-N*

1.1

180.0

2.0

NONBON 
Additional Force Field Parameters for TPR

Remark line goes here

MASS

BOND

ANGLE

C - CT-N* $68.100 \quad 106.510$ same as ca-c3-nc, penalty score $=2.5$

DIHE

IMPROPER

C $-\mathrm{CT}-\mathrm{N}-\mathrm{CT}$

1.1

180.0

2.0

Using the default

value

$\mathrm{CT}-\mathrm{N}-\mathrm{C}-\mathrm{O}$

1.1

180.0

2.0

Using the default

value

$\mathrm{N}^{*}-\mathrm{NA}-\mathrm{C}-\mathrm{O}$

1.1

180.0

2.0

Using the default

value

C -C -NA-H

1.1

180.0

2.0

Using the default

value

CM-NA-C -O

1.1

180.0

2.0

Using the default

value

1.1

180.0

2.0

Using the default

value

NONBON 
Additional Force Field Parameters for QPC

Remark line goes here

MASS

BOND

CA-OS $\quad 450.00 \quad 1.364$

CA-N* $436.00 \quad 1.374$

ANGLE

C - CT-CA $63.700 \quad 111.010$ same as c -c3-ca, penalty score $=0.0$

CA-CA-N* $70.000 \quad 121.500$

CA-CA-OS $70.000 \quad 120.000$

CA-N*-CA $70.000 \quad 105.400$

$\begin{array}{lll}\mathrm{H} 4-\mathrm{CA}-\mathrm{N}^{*} \quad 50.000 & 119.100\end{array}$

DIHE

$\begin{array}{lllll}\text { CA-CA-N*-CA } & 4 & 6.600 & 180.000 & 2.000 \\ \text { H4-CA-N*-CA } & 4 & 6.600 & 180.000 & 2.000\end{array}$

IMPROPER

C $-\mathrm{CT}-\mathrm{N}-\mathrm{CT}$

1.0

180.0

2.0

$\mathrm{CT}-\mathrm{N}-\mathrm{C}-\mathrm{O}$

10.5

180.0

2.0

CA-CA-CA-CA

1.1

180.0

2.0

CA-CA-CA-HA

1.1

180.0

2.0

CA-CA-CA-OS

1.1

180.0

2.0

CA-CA-CA-N*

1.1

180.0

2.0 
NONBON 
Additional Force Field Parameters for QPN

Remark line goes here

MASS

ОТ $16.000 \quad 0.465$

NX $14.010 \quad 0.530$

BOND

CA-OT $\quad 480.00 \quad 1.240$

CA-NX $\quad 436.00 \quad 1.374$

ANGLE

C - CT-CA $63.700 \quad 111.010$ same as c - 3 - - ca, penalty score $=0.0$

$\begin{array}{lll}\text { CA-CA-NX } \quad 70.000 & 121.200\end{array}$

$\begin{array}{lll}\text { CA-CA-OT } \quad 80.000 & 125.000\end{array}$

CA-NX-CA $\quad 70.000 \quad 105.400$

$\begin{array}{lll}H 4-C A-N X \quad 50.000 & 119.100\end{array}$

DIHE

$\begin{array}{lllll}\text { CA-CA-NX-CA } & 4 & 6.600 & 180.000 & 2.000 \\ \text { H4-CA-NX-CA } & 4 & 6.600 & 180.000 & 2.000\end{array}$

IMPROPER

C $-\mathrm{CT}-\mathrm{N}-\mathrm{CT}$

1.0

180.0

2.0

CT-N - C - O

10.5

180.0

2.0

CA-CA-CA-CA

1.1

180.0

2.0

CA-CA-CA-HA

1.1

180.0

2.0 


$\begin{array}{llll}\text { CA-CA-CA-OT } & 1.1 & 180.0 & 2.0 \\ \text { CA-CA-CA-NX } & 1.1 & 180.0 & 2.0 \\ \text { CA-HA-CA-NX } & 1.1 & 180.0 & 2.0\end{array}$

NONBON

\begin{tabular}{|c|c|}
\hline OT & 1.6837 \\
\hline$N X$ & 1.8240 \\
\hline
\end{tabular}




\section{Additional Force Field Parameters for $\mathrm{Cu}$}

Remark line goes here

MASS

Cu $63.010 \quad 0.000$

BOND

ANGLE

DIHE

IMPROPER

NONBON

$\begin{array}{lll}\mathrm{Cu} & 2.2100 & 0.1729\end{array}$ 
All other additional Force Field Parameters involved by $\mathrm{Cu}$

Remark line goes here

MASS

BOND

$\begin{array}{llll}\text { CU-N* } & 48.51 & 1.999 & \text { calculated for } \mathrm{Cu}-\mathrm{N}^{*} \\ \text { CU-OS } & 46.58 & 1.953 & \text { calculated for } \mathrm{Cu}-\mathrm{OS} \\ \text { CU-NX } & 48.46 & 1.999 & \text { calculated for } \mathrm{Cu}-\mathrm{NX} \\ \text { CU-OT } & 46.63 & 1.953 & \text { calculated for Cu-OT }\end{array}$

ANGLE

CA-N*-Cu $19.508 \quad 120.151$ Calculated with empirical approach for CA-N*-

$\mathrm{Cu}$

$\mathrm{N}^{*}$-CU-OT $42.795 \quad 96.602$ Calculated with empirical approach for $\mathrm{N}^{*}-\mathrm{Cu}-$
OT

N*-Cu-NX $31.034 \quad 179.595$ Calculated with empirical approach for $\mathrm{N}^{*}$-Cu$\mathrm{NX}$

OS-Cu-OT $31.742 \quad 179.781$ Calculated with empirical approach for OS-CuOT

$\mathrm{N}^{*}$-Cu-OS $45.996 \quad 83.609$ Calculated with empirical approach for $\mathrm{N}^{*}-\mathrm{Cu}$ OS

NX-Cu-OS $42.860 \quad 96.272$ Calculated with empirical approach for NX-CuOS

CA-OS-Cu 20.327 112.388 Calculated with empirical approach for CA-OS$\mathrm{Cu}$

CA-NX-Cu $19.503 \quad 120.158$ Calculated with empirical approach for CA-NX$\mathrm{Cu}$

NX-Cu-OT $46.021 \quad 83.518$ Calculated with empirical approach for NX-CuOT

CA-OT-Cu 20.327 112.429 Calculated with empirical approach for CA-OT$\mathrm{Cu}$ 
DIHE

\begin{tabular}{|c|c|c|c|c|c|}
\hline $\begin{array}{l}\mathrm{H} 4-\mathrm{CA}-\mathrm{N}^{*}-\mathrm{Cu} \\
\mathrm{X} \text {, penalty }\end{array}$ & $\begin{array}{c}2 \\
\text { score }=\end{array}$ & $\begin{array}{c}9.600 \\
0.0\end{array}$ & 180.000 & 2.000 & same as X-ca-nd- \\
\hline $\begin{array}{l}\mathrm{CA}-\mathrm{CA}-\mathrm{N}^{*}-\mathrm{Cu} \\
\mathrm{X} \text {, penalty }\end{array}$ & $\begin{array}{c}2 \\
\text { score }=\end{array}$ & $\begin{array}{c}9.600 \\
0.0\end{array}$ & 180.000 & 2.000 & same as X-ca-nd- \\
\hline $\mathrm{OT}-\mathrm{Cu}-\mathrm{N}^{*}-\mathrm{CA}$ & 1 & 0.000 & 0.000 & 2.000 & ATTN, need revision \\
\hline $\mathrm{NX}-\mathrm{Cu}-\mathrm{N}^{*}-\mathrm{CA}$ & 1 & 0.000 & 0.000 & 2.000 & ATTN, need revision \\
\hline $\mathrm{N}^{*}-\mathrm{CU}-\mathrm{OT}-\mathrm{CA}$ & 1 & 0.000 & 0.000 & 2.000 & ATTN, need revision \\
\hline $\mathrm{N}^{*}-\mathrm{CU}-\mathrm{NX}-\mathrm{CA}$ & 1 & 0.000 & 0.000 & 2.000 & ATTN, need revision \\
\hline $\begin{array}{l}C A-C A-O S-C u \\
x \text {, penalty }\end{array}$ & $\begin{array}{c}2 \\
\text { score }=\end{array}$ & $\begin{array}{c}1.800 \\
0.0\end{array}$ & 180.000 & 2.000 & same as X -ca-os- \\
\hline OS-CU-OT-CA & 1 & 0.000 & 0.000 & 2.000 & ATTN, need revision \\
\hline $\mathrm{OS}-\mathrm{CU}-\mathrm{N}^{*}-\mathrm{CA}$ & 1 & 0.000 & 0.000 & 2.000 & ATTN, need revision \\
\hline OS-CU-NX-CA & 1 & 0.000 & 0.000 & 2.000 & ATTN, need revision \\
\hline OT-CU-OS-CA & 1 & 0.000 & 0.000 & 2.000 & ATTN, need revision \\
\hline $\mathrm{N}^{*}-\mathrm{CU}-\mathrm{OS}-\mathrm{CA}$ & 1 & 0.000 & 0.000 & 2.000 & ATTN, need revision \\
\hline$N X-C u-O S-C A$ & 1 & 0.000 & 0.000 & 2.000 & ATTN, need revision \\
\hline $\begin{array}{l}\mathrm{H} 4-\mathrm{CA}-\mathrm{NX}-\mathrm{Cu} \\
\mathrm{X} \text {, penalty }\end{array}$ & $\begin{array}{c}2 \\
\text { score }=\end{array}$ & $\begin{array}{c}9.600 \\
0.0\end{array}$ & 180.000 & 2.000 & same as X-ca-nd- \\
\hline $\begin{array}{l}C A-C A-N X-C u \\
X \text {, penalty }\end{array}$ & $\begin{array}{c}2 \\
\text { score }=\end{array}$ & $\begin{array}{c}9.600 \\
0.0\end{array}$ & 180.000 & 2.000 & same as X - ca-nd- \\
\hline $\begin{array}{l}\mathrm{CA}-\mathrm{CA}-\mathrm{OT}-\mathrm{Cu} \\
\mathrm{X} \text {, penalty }\end{array}$ & $\begin{array}{c}2 \\
\text { score }=\end{array}$ & $\begin{array}{c}1.800 \\
0.0\end{array}$ & 180.000 & 2.000 & same as X -ca-os- \\
\hline OT-CU-NX-CA & 1 & 0.000 & 0.000 & 2.000 & ATTN, need revision \\
\hline $\mathrm{NX}-\mathrm{CU}-\mathrm{OT}-\mathrm{CA}$ & 1 & 0.000 & 0.000 & 2.000 & ATTN, need revision \\
\hline
\end{tabular}

IMPROPER

NONBON 


\section{References}

1. Wittung, P.; Eriksson, M.; Lyng, R.; Nielsen, P. E.; Norden, B., Induced Chirality in PNAPNA Duplexes. Journal of the American Chemical Society 1995, 117 (41), 10167-10173.

2. Wittung, P.; Nielsen, P. E.; Buchardt, O.; Egholm, M.; Norde'n, B., DNA-like double helix formed by peptide nucleic acid. Nature 1994, 368 (6471), 561-563.

3. Watson, R. M.; Skorik, Y. A.; Patra, G. K.; Achim, C., Influence of Metal Coordination on the Mismatch Tolerance of Ligand-Modified PNA Duplexes. Journal of the American Chemical Society 2005, 127 (42), 14628-14639.

4. Neese, F., The ORCA program system. WIREs Computational Molecular Science 2012, 2 (1), 73-78.

5. Neese, F., Software update: the ORCA program system, version 4.0. WIREs Computational Molecular Science 2018, 8 (1), e1327.

6. Humphrey, W.; Dalke, A.; Schulten, K., VMD: visual molecular dynamics. Journal of molecular graphics 1996, 14 (1), 33-38. 